\title{
Changes in Frequency of Large Precipitation Accumulations over Land in a Warming Climate from the CESM Large Ensemble: The Roles of Moisture, Circulation, and Duration ${ }^{\mathscr{O}}$
}

\author{
Jesse NorRis, GANG CHEN, AND J. DAVID NEELIN \\ Department of Atmospheric and Oceanic Sciences, University of California, Los Angeles, Los Angeles, California
}

(Manuscript received 10 September 2018, in final form 24 May 2019)

\begin{abstract}
Projected changes in the frequency of major precipitation accumulations (hundreds of millimeters), integrated over rainfall events, over land in the late twenty-first century are analyzed in the Community Earth System Model (CESM) Large Ensemble, based on the RCP8.5 scenario. Accumulation sizes are sorted by the local average recurrence interval (ARI), ranging from 0.1 to 100 years, for the current and projected latetwenty-first-century climates separately. For all ARIs, the frequency of exceedance of the given accumulation size increases in the future climate almost everywhere, especially for the largest accumulations, with the 100 -yr accumulation becoming about 3 times more frequent, averaged over the global land area. The moisture budget allows the impacts of individual factors-moisture, circulation, and event duration-to be isolated. In the tropics, both moisture and circulation cause large future increases, enhancing the 100-yr accumulation by $23 \%$ and $13 \%$ (average over tropical land), and are individually responsible for making the current-climate 100 -yr accumulation 2.7 times and 1.8 times more frequent, but effects of shorter durations slightly offset these effects. In the midlatitudes, large accumulations become about $5 \%$ longer in duration, but are predominantly controlled by enhanced moisture, with the 100-yr accumulation (land average) becoming 2.4 times more frequent, and 2.2 times more frequent due to moisture increases alone. In some monsoon-affected regions, the 100 -yr accumulation becomes more than 5 times as frequent, where circulation changes are the most impactful factor. These projections indicate that changing duration of events is a relatively minor effect on changing accumulations, their future enhancement being dominated by enhanced intensity (the combination of moisture and circulation).
\end{abstract}

\section{Introduction}

In a warming climate, extreme precipitation is projected to increase over most of the globe, which may be attributed to an enhancement of saturation vapor humidity, depending on the Clausius-Clapeyron relation (e.g., Trenberth 1999; Allen and Ingram 2002), but in some regions may also be enhanced by greater dynamical forcing (e.g., Emori and Brown 2005; Pfahl et al. 2017; Tandon et al. 2018; Norris et al. 2019). Analysis of projected changes to extremes has typically been based on daily precipitation totals (e.g., Emori and Brown 2005; Pall et al. 2007; Kharin et al. 2013; Pendergrass and

\footnotetext{
Supplemental information related to this paper is available at the Journals Online website: https://doi.org/10.1175/JCLI-D-180600.s1.
}

Corresponding author: Jesse Norris, jessenorris@ucla.edu
Hartmann 2014a,b; Donat et al. 2016; Pfahl et al. 2017), upon which our current understanding of the global patterns is based. Also of direct relevance to societal impacts, in particular flooding events, is how the large accumulations will change. We distinguish an event "accumulation," the integration of precipitation over the duration of a rainfall event, from a "total" over a fixed period.

Although the accumulation is not the only important metric for flooding - for example $100 \mathrm{~mm}$ of rainfall in $1 \mathrm{~h}$ will lead to worse flooding than $100 \mathrm{~mm}$ over a few days-the future changes to accumulations, as opposed to fixed-period totals, have received relatively little attention in the literature, and study of these events may identify unique aspects of the changing character of precipitation. The greatest accumulations may result from precipitation events longer than 1 day. For example, extreme monsoon rains may continue for multiple days in succession with little respite, and the largest 
accumulations in subtropical/midlatitude locations may result from successive frontal systems over multiple days. In these cases, although precipitation may not be continuous, the accumulation size over successive days is highly relevant for flooding.

In recent years, accumulations on the order of $1000 \mathrm{~mm}$ have occurred around the globe, for example, after the 2017 hurricane season in Texas, Florida, and Puerto Rico, and after the extreme monsoon rains over India and Bangladesh in 2017. Although this magnitude of accumulation is generally restricted to the tropics or areas prone to tropical cyclone landfall, subtropical and midlatitude regions have recently received accumulations from extratropical cyclones that are also of extreme impact and locally almost unprecedented. For example, accumulations on the order of $100 \mathrm{~mm}$ were recorded in the winter of 2013/14 in the United Kingdom and in the winter of 2016/17 in California, and in both of these cases the events were embedded within winters that were among the wettest on record.

Historical observational datasets indicate that precipitation accumulations (Martinez-Villalobos and Neelin 2018) and daily or multiday totals (Alexander et al. 2006) increased through the late twentieth century. The observed rate of increase of annual-maximum daily precipitation is significantly positively correlated with the observed rate of global-mean warming (Westra et al. 2013), illustrating the importance of greater moisture capacity in a warmer climate for increasing accumulations. However, increases in extremes may be greater than or less than the prediction based on Clausius-Clapeyron, depending on changes to atmospheric circulation (Emori and Brown 2005; Tandon et al. 2018; Chen et al. 2019; Norris et al. 2019). For accumulations, changes to the duration of events may also play a role (Dwyer and O'Gorman 2017; Martinez-Villalobos and Neelin 2018). For flood-risk mitigation, it is highly desirable to project how much more frequent accumulations of a given magnitude, for example, the 100 -yr event, will become over land in a future warmer climate, and to understand the contributions of the different factors affecting the accumulations to those changes.

Changes in the frequency of accumulations depend on the probability density function (PDF) of accumulation. The PDF of accumulation is maximized at low values and slowly decreases over a power-law range up to some cutoff beyond which the frequency drops rapidly, as demonstrated by climate models (Neelin et al. 2017) and observations (Peters et al. 2010; Stechmann and Neelin 2014). Neelin et al. (2017) produced climate projections with the Community Earth System Model (CESM) whereby the frequency of relatively low accumulations was not greatly affected, but the cutoff was increased, so that the frequency of events above the current-climate cutoff vastly increased. A similar projection was evident in the results of Kao and Ganguly (2011), who performed a percentile regression for precipitation totals over various intervals ranging from 5 to $200 \mathrm{~h}$. In their analysis, for all future climate scenarios and for all intervals, the frequency of the most extreme totals increased in a warmer climate. Because some regions exhibit greater increases in cutoff than others (Neelin et al. 2017), the question arises, what controls the local increase in cutoff with warming? And how much increase in cutoff can be explained by individual processes?

Precipitation accumulation is the product of duration and intensity of rainfall events. Because precipitation intensity almost universally increases with warming (Pendergrass et al. 2017; Pfahl et al. 2017; Tandon et al. 2018; Norris et al. 2019), the same accumulation may be achieved with a shorter duration, but climate models suggest that any decreases in duration will not nearly compensate the increases of intensity. In particular, phase 5 of the Coupled Model Intercomparison Project (CMIP5) models project a decrease in the duration of midlatitude precipitation of approximately $1 \%$ per degree of global warming, due to stronger westerlies, but compared with the projected increase in precipitation intensity (about $7 \%$ per degree of warming, assuming no change in atmospheric circulation), this suggests that the changing intensity is the most important factor for changing accumulations (Dwyer and O'Gorman 2017). That study's analysis was based on percentiles of precipitation intensity, which may not identify the greatest accumulations. In particular, events of great intensity may not register as significant accumulations if they are too short-lived. Similarly, events of great duration may not register if they are of low intensity. The current study focuses on the events that deliver large accumulations and breaks them down into duration and intensity. Following Chen et al. (2019) and Norris et al. (2019), intensity may be further broken down by the moisture budget equation into vertical moisture transport, horizontal moisture advection, and moisture storage. Those studies showed that, for extremes of precipitation rate, vertical moisture transport is the dominant term, so that in theory an accumulation should be approximately the product of duration, gross moisture stratification, and low-level mass convergence.

This study analyzes the moisture budget equation for projected changes to precipitation accumulations using an ensemble of climate projections. A similar analysis was recently performed for projected changes to various percentiles of instantaneous precipitation (Chen et al. 2019; Norris et al. 2019). The extra component contributing to 
accumulations is the duration of events, which we consider in more detail in this study. By comparing results between the present and future climates, we project how much more frequent major accumulations will become, and how much more frequent they would become if moisture, circulation, or duration alone were to change.

\section{Data and methodology}

\section{a. The CESM Large Ensemble}

The CESM Large Ensemble (Kay et al. 2015) was analyzed for both the end-of-twentieth (1990-2005, hereafter E20) and a projected late-twenty-first (2071-80, hereafter E21) century climate. The future climate was simulated assuming the RCP8.5 scenario, in which greenhouse gas emissions continue to increase throughout the twenty-first century. This is an extreme scenario, and so our results are presented with the caveat that, for lower emissions scenarios, changes will likely be less than presented in this study. The Large Ensemble was analyzed using 6-hourly output in order to measure accumulations (i.e., daily data would be too coarse in time), but selected hourly data were also used to validate the 6-hourly data (section 2d). For 6-hourly data, the Large Ensemble output consists of 42 members for E20 and 40 members for E21. Each member has distinct initial conditions, so that the difference between the members is due to internal variability, but the ensemble mean is an estimate of the response to external forcing.

The analysis in this study is on major accumulation sizes that are exceeded once every $e$ years, where $e$ ranges from 0.1 up to 100 years. Therefore, a dataset of several hundred years was required. Because each member contains just 16 and 10 years of output for E20 and E21 using the 6-hourly data, the members were aggregated to generate a single distribution of events. To further smooth the analysis, the distributions were aggregated over the $3 \times 3$ grid points surrounding each grid point to generate a single dataset of 6048 years for E20 (16 years $\times$ 42 members $\times 9$ grid points) and 3600 years for E21 (10 years $\times 40$ members $\times 9$ grid points). The much greater dataset for E20 than E21 makes no discernable difference to the results presented, that is, reducing the E20 dataset to 10 years and 40 members, as in E21, leads to almost identical results (not shown), but we present results using the full dataset for E20 for completeness sake. At each grid point, these aggregated datasets were analyzed as follows in the sections $2 \mathrm{~b}$ and $2 \mathrm{c}$.

\section{b. Precipitation accumulations}

An accumulation is defined as the integral over time of consecutive time slices with $>0.5 \mathrm{~mm} \mathrm{~h}^{-1}$ precipitation,

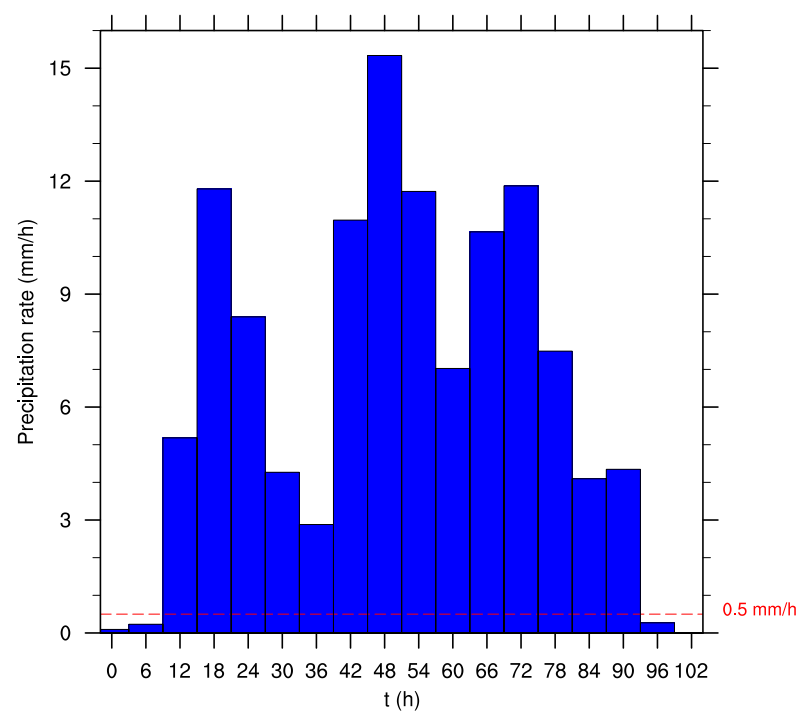

FIG. 1. An example of an extreme precipitation accumulation recorded from the CESM Large Ensemble. The blue bars are precipitation rate every $6 \mathrm{~h}$, which is assumed to be constant during the $6 \mathrm{~h}$ surrounding each output time. An accumulation is considered to begin when the rate first exceeds $0.5 \mathrm{~mm} \mathrm{~h}^{-1}$ (dashed red line) and end when the rate first drops below $0.5 \mathrm{~mm} \mathrm{~h}^{-1}$, hence this accumulation lasts from 12 to $90 \mathrm{~h}$. The accumulation size is calculated by summing each precipitation rate during the accumulation and multiplying by the output frequency $(6 \mathrm{~h})$. In this example, the sum of precipitation rates is 116 , hence the total accumulation is $696 \mathrm{~mm}$. The duration is calculated as $84 \mathrm{~h}$ (14 time slices, multiplied by $6 \mathrm{~h})$.

similarly to Neelin et al. (2017). As soon as the precipitation rate drops below $0.5 \mathrm{~mm} \mathrm{~h}^{-1}$, the event is considered to be over, and then the next time slice with $>0.5 \mathrm{~mm} \mathrm{~h}^{-1}$ precipitation marks the beginning of the next accumulation. An extreme example recorded by the CESM Large Ensemble is shown in Fig. 1 in which an accumulation of $696 \mathrm{~mm}$ is recorded over $84 \mathrm{~h}$.

\section{c. Identification of accumulation sizes for various ARIs}

The identified accumulations during the aggregated dataset were stored in ascending order and used to identify accumulation sizes for a range of average recurrence intervals (ARIs). ARI is defined as the average or expected value of the period between exceedances of a given rainfall amount (Parzybok et al. 2011). A set of ARIs of accumulation size was established using the following formula:

$$
e_{i}=10^{-1+0.25 i} \text { for } i=0,1, \ldots, 12,
$$

where $e_{i}$ denotes the number of years $e$ of the $i$ th ARI. This formula was designed to generate a set of ARIs increasing exponentially from 0.1 (approximately 1 month) 
TABLE 1. Average recurrence intervals (ARIs) and ARI ranges in years calculated by $e_{i}=10^{-1+0.25 i}$, used for the analysis in this study. Parameter $e_{i}$ denotes the ARI that is used for annotation of figures and for the $x$ axis in figures shown as a function of ARI. The $e$-yr accumulation is given by the $Y /$ eth (rounding to the nearest integer) greatest accumulation during the full distribution of events, where $Y$ is the aggregated number of years. The $e_{i}$-yr accumulation is calculated by conditionally averaging the probability distribution between the $e_{i-1 / 2}$-yr and $e_{i+1 / 2}$-yr accumulations. The $e_{i-1 / 2}$ and $e_{i+1 / 2}$ are calculated by replacing $i$ with $i-1 / 2$ and $i+1 / 2$ in the above equation.

\begin{tabular}{llcccccccccccc}
\hline \hline \multicolumn{1}{c}{$\boldsymbol{c}$} & 0 & 1 & 2 & 3 & 4 & 5 & 6 & 7 & 8 & 9 & 10 & 11 & 12 \\
\hline$e_{i}$ & 0.10 & 0.18 & 0.32 & 0.56 & 1.0 & 1.8 & 3.2 & 5.6 & 10 & 18 & 32 & 56 & 100 \\
$e_{i-1 / 2}$ & 0.075 & 0.13 & 0.24 & 0.42 & 0.75 & 1.3 & 2.4 & 4.2 & 7.5 & 13 & 24 & 42 & 75 \\
$e_{i+1 / 2}$ & 0.13 & 0.24 & 0.42 & 0.75 & 1.3 & 2.4 & 4.2 & 7.5 & 13 & 24 & 42 & 75 & 133 \\
\hline
\end{tabular}

to 100 years, and including 1 and 10 years. The accumulation associated with the $e$-yr ARI was then given by the Y/eth (rounding to the nearest integer) greatest accumulation during the full dataset, where $Y$ is the aggregated number of years. This method implies that the $i$ th accumulation is given by the value which is exceeded on average every $e_{i}$ years. For example, for E20, which has a 6048-yr dataset at each grid point, the 60480 th, 6048th, 605th, and 60th greatest accumulations give the 0.1-, 1-, 10-, and 100-yr accumulations, respectively.

To increase the sample size for each ARI, the $e_{i}$-yr accumulation was calculated by averaging over the probability distribution of precipitation accumulation between the $e_{i-1 / 2}$-yr and $e_{i+1 / 2}$-yr ARIs, where $e_{i-1 / 2}$ and $e_{i+1 / 2}$ are obtained by replacing $i$ by $i-1 / 2$ and $i+1 / 2$, respectively, in (1). The method is analogous to the analysis of instantaneous precipitation in Chen et al. (2019) and Norris et al. (2019), with precipitation percentile in those studies replaced by ARI in this study. The full set of ARIs given by (1) and the ARI ranges used to calculate the accumulation for each ARI are given in Table 1.

Note that this method leaves missing data for certain ARIs at certain grid points if there are insufficient accumulations in the dataset, which is an issue in the subtropical dry zones. For example, in E20 if there are fewer than 60480 accumulations at some grid point during the 6048-yr aggregated dataset, then the 0.1-yr accumulation does not exist. Therefore, at some grid points there is an $e_{i}$-yr ARI for some $i$ below which no accumulations are identified. Data were set to missing for the $e_{i}$-yr ARI if the $e_{i-1 / 2}$-yr accumulation did not exist, that is, if there were fewer than $Y / 10^{-1+0.25(i-0.5)}$ accumulations (rounded to the nearest integer) in the dataset, where $Y$ is the aggregated number of years, because this implies that the $e_{i}$-yr accumulation could not be calculated by averaging between the $(i-1 / 2)$ th and $(i+1 / 2)$ th ARIs. This missing data will be evident in certain figures in this paper, but mostly just for the 0.1-yr ARI and does not significantly restrict the analysis.

\section{d. Observational and model datasets used for evaluation}

To verify that the 6-hourly data are of sufficient temporal resolution to represent accumulations, hourly data from the CESM Large Ensemble were also utilized. The hourly data are of the same simulations as the 6-hourly data, but available for the historic climate just for two members from 1920 to 2005 , which were aggregated to form a single 1548-yr (86 years $\times 2$ members $\times 9$ grid points) dataset of hourly precipitation at each grid point. The much shorter aggregated dataset than for 6-hourly data is the reason that hourly data were not used for the analysis in this study, but for relatively short ARIs (up to 10 years) they may be used to evaluate the 6-hourly data.

Satellite rainfall estimates from the Tropical Rainfall Measuring Mission (TRMM) 3B42 (Huffman et al. 2007) were also utilized, available between $50^{\circ} \mathrm{S}$ and $50^{\circ} \mathrm{N}$ and from 1998 through 2017 (hence an aggregated dataset of 20 years $\times 9$ grid points $=180$ years $)$ at $0.25^{\circ} \times 0.25^{\circ}$ latitude-longitude grid spacing and 3-hourly temporal resolution. Both the hourly CESM and 3-hourly TRMM data were subject to the same analysis described in sections $2 \mathrm{~b}$ and $2 \mathrm{c}$ and compared to the 6-hourly CESM accumulations in E20 for ARIs up to 10 years. The analysis for TRMM was performed on its native grid and then the output bilinearly interpolated onto the CESM grid for comparison.

\section{Moisture budget}

\section{a. Moisture budget equation}

To identify factors affecting changes in the frequency of accumulations for different ARIs, the moisture budget equation was analyzed (e.g., Seager and Henderson 2013), which for instantaneous precipitation states that precipitation minus evaporation is equal to moisture flux convergence minus the storage of column water vapor:

$$
P-E=-\frac{1}{g} \nabla \cdot \int_{0}^{p_{s}} \mathbf{v} q d p-\frac{1}{g} \frac{\partial}{\partial t} \int_{0}^{p_{s}} q d p
$$


where $P$ is precipitation, $E$ is evaporation, $\mathbf{v}$ is the horizontal wind vector, $q$ is the water vapor mixing ratio, $p_{s}$ is surface pressure, and $g$ is acceleration due to gravity. Discretizing the equation and denoting the massweighted vertical integral of some variable $X$ as $\{X\}=$ $1 / g \sum_{k}\left(X_{k} d p_{k}\right)$, where $X_{k}$ is $X$ at the $k$ th model level and $d p_{k}$ is the pressure difference between the bottom and top of the $k$ th model level, it may be written as

$$
P-E=-\nabla \cdot\{\mathbf{v} q\}-\frac{\partial}{\partial t}\{q\} .
$$

Following Chen et al. (2019) and Norris et al. (2019), we define the dry-air mass convergence $C_{k}$ at the $k$ th model level:

$$
C_{k}=-\frac{1}{g} \nabla \cdot\left(\mathbf{v}_{k} d p_{k}\right)
$$

so that the equation becomes

$$
P-E=\sum_{k}\left(q_{k} C_{k}\right)-\{\mathbf{v} \cdot \nabla q\}-\frac{\partial}{\partial t}\{q\} .
$$

We may multiply both sides of the equation by the duration of the event $D$, defined as the number of time slices contributing to the accumulation multiplied by the output frequency $(6 \mathrm{~h})$. For example, in Fig. 1, the duration of the accumulation is $84 \mathrm{~h}$. Hence, we obtain

$$
P_{\mathrm{acc}}=D \bar{P}=D \sum_{k} \overline{q_{k} C_{k}}-D \overline{\{\mathbf{v} \cdot \nabla q\}}-D \overline{\frac{\partial}{\partial t}\{q\}}+D \bar{E},
$$

where overbars denote the time mean during an accumulation. To simplify the equation, we assume that $\bar{E}$ and $\overline{\partial / \partial t\{q\}}$ are both negligible. The neglect of $\bar{E}$ is because we assume that precipitation is vastly greater than evaporation for extreme events, and of $\overline{\partial / \partial t\{q\}}$ because we assume that the moisture content at the beginning of the event is approximately equal to the moisture content at the end of the event. Then, we assume that the eventmean vertical moisture transport at the $k$ th model level $\overline{q_{k} C_{k}}$ is approximately equal to the product of the eventmean moisture and event-mean mass convergence, that is, $\overline{q_{k} C_{k}} \approx \overline{q_{k}} \overline{C_{k}}$, thus neglecting covariance of $q_{k}$ and $C_{k}$ during the event. Finally, although accumulated horizontal moisture advection $D \overline{\{\mathbf{v} \cdot \nabla q\}}$ may not be negligible, we may expect that the accumulated vertical moisture transport $D \sum_{k}\left(\overline{q_{k}} \overline{C_{k}}\right)$ is the dominant term. This assumption is based on a similar finding for percentiles of precipitation rate in Chen et al. (2019) and Norris et al. (2019). If all these approximations are reasonable, then the approximation for accumulated precipitation is simply

$$
P_{\mathrm{acc}} \approx D \sum_{k}\left(\overline{q_{k}} \overline{C_{k}}\right)
$$

that is, at a given grid point, the product of duration and the vertical profiles of event-mean moisture and mass convergence gives the accumulated precipitation. The vertical transport $D \sum_{k}\left(\overline{q_{k}} \overline{C_{k}}\right)$ and horizontal advection $-D \overline{\{\mathbf{v} \cdot \nabla q\}}$ terms are compared for accumulations in the appendix.

\section{b. Thermodynamic and dynamic contributions to accumulations}

If vertical moisture transport can be separated into the product of vertically integrated variables representing moisture and mass convergence separately, then the right-hand side of (4) is the product of (i) duration, (ii) moisture, and (iii) mass convergence. This analysis is achieved by use of the same variables $M_{*}$ and $C_{*}$ defined in Chen et al. (2019) and Norris et al. (2019), representing gross moisture stratification and strength of low-level convergence, respectively. Specifically, we define a weighting function $\tilde{C}_{k}^{e}$, such that at the $k$ th model level for a given event

$$
\overline{C_{k}}=C_{*} \tilde{C}_{k},
$$

where the low-level convergence is

$$
C_{*}=\operatorname{SIGN}\left[\sum_{k}\left(\overline{q_{k}} \overline{C_{k}}\right)\right] \operatorname{STD}\left(\overline{C_{k}}\right),
$$

where SIGN $\left[\sum_{k}\left(\overline{q_{k}} \overline{C_{k}}\right)\right]$ is the sign of vertical moisture transport $\sum_{k}\left(\overline{q_{k}} \overline{C_{k}}\right)$, and $\operatorname{STD}\left(\overline{C_{k}}\right)$ is the standard deviation of $\overline{C_{k}}$ in the vertical. Hence, given the typical structure of decreasing humidity with height, $C_{*}$ is positive where there is low-level convergence and upper-level divergence and negative where there is low-level divergence and upper-level convergence.

We then define the gross moisture stratification:

$$
M_{*}=\sum_{k}\left(\overline{q_{k}} \tilde{C}_{k}\right)=\frac{\sum_{k}\left(\overline{q_{k}} \overline{C_{k}}\right)}{C_{*}},
$$

where $M_{*}$ is positive definite, because the sign of $C_{*}$ is by definition the same as the sign of vertical moisture transport $\sum_{k}\left(\overline{q_{k}} \overline{C_{k}}\right)$. Therefore, vertical moisture transport is given by

$$
\sum_{k}\left(\overline{q_{k}} \overline{C_{k}}\right)=M_{*} C_{*} .
$$

Hence, if the vertical transport term [the right-hand side of (4)] is the dominant term for a precipitation accumulation, then a given accumulation is the product of three vertically integrated variables, representing gross 

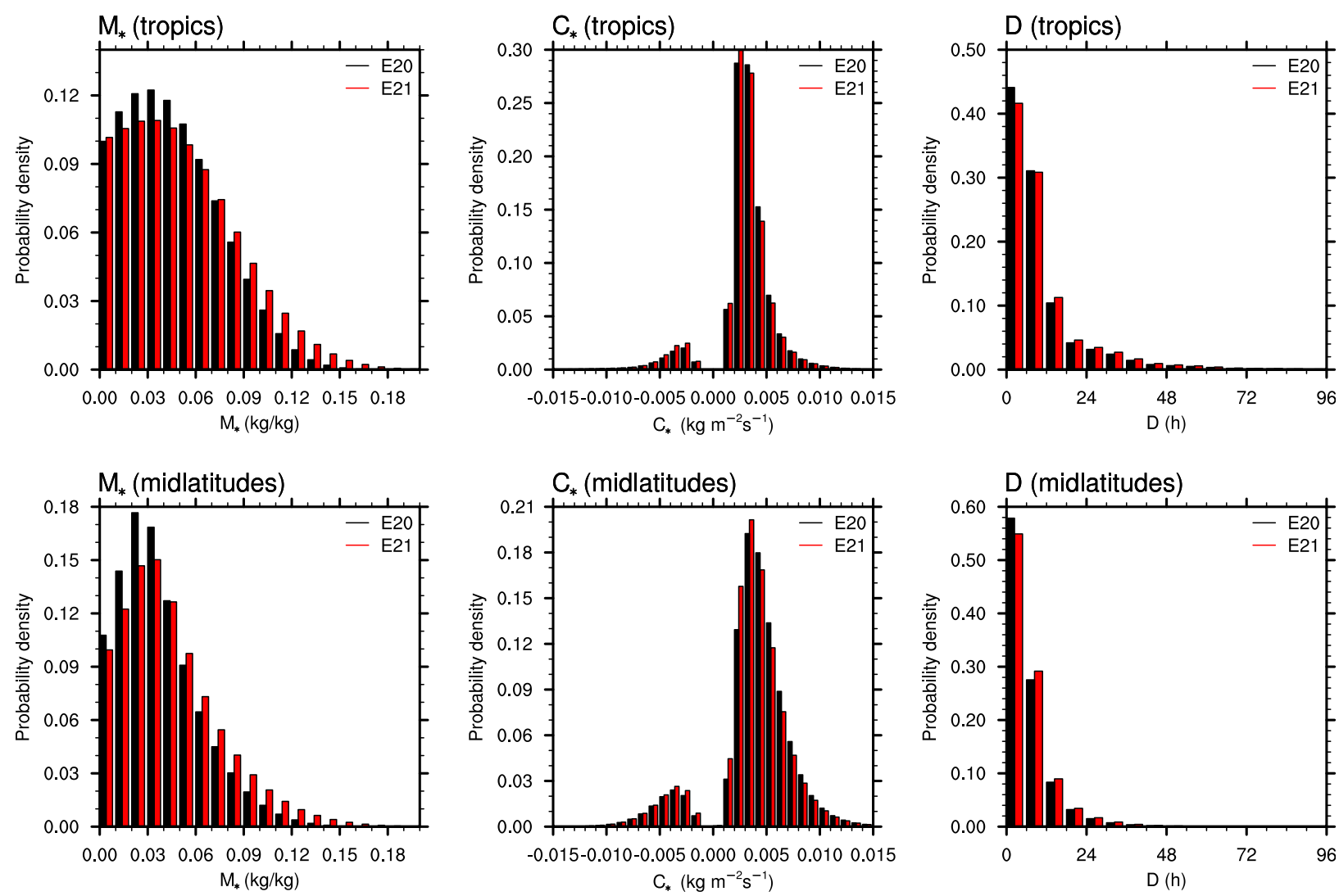

FIG. 2. Histograms of the PDFs of $M_{*}, C_{*}$, and $D$, representing moisture, circulation, and duration of events, respectively, in E20 (black) and E21 (red). The PDF is calculated at each grid point of the given variable from all events registering as accumulations (section 2b). Then the PDFs are averaged over all land grid points (top) between $15^{\circ} \mathrm{S}$ and $15^{\circ} \mathrm{N}$ and (bottom) between $40^{\circ}$ and $50^{\circ}$ in each hemisphere.

moisture stratification, low-level mass convergence, and duration:

$$
P_{\mathrm{acc}} \approx M_{*} C_{*} D .
$$

The distributions of $M_{*}, C_{*}$, and $D$ are shown in Fig. 2 for E20 (black bars) and E21 (red bars), averaged over the tropics (top panels) and midlatitudes (bottom panels). These are the values obtained during all events that register as accumulations (section $2 b$ ). As described above, $M_{*}$, representing moisture, is necessarily positive and its tail drops off more slowly in the tropics than midlatitudes (left column), illustrating the relative rarity of high moisture levels in the midlatitudes (i.e., just during atmospheric river events). Unsurprisingly, the vast majority of events experience positive $C_{*}$, that is, low-level convergence when averaged over the event, but there is a small proportion of negative values, indicating some accumulations are generated under eventmean divergent conditions (center column). In E21, in both the tropics and midlatitudes, for $M_{*}$ there is a lower frequency of low values and a greater frequency of high values than in E20, whereas for $C_{*}$ there is a greater frequency of low/negative values and a lower frequency of high values. These changes to the PDFs of $M_{*}$ and $C_{*}$ illustrate the moister future atmosphere but with a weakened mean global circulation.

Duration illustrates that almost half events in the tropics are just $6 \mathrm{~h}$ (a single time slice) and slightly more than half in the midlatitudes (right column). Duration is generally greater in the tropics than midlatitudes, with about $10 \%$ of events $>24 \mathrm{~h}$ in the tropics and about $3 \%$ in the midlatitudes. In E21, both the tropics and midlatitudes experience a smaller fraction of 6-h events, and a greater fraction of longer events. It is possible that this effect arises from the implementation of a minimum threshold of precipitation rate $\left(0.5 \mathrm{~mm} \mathrm{~h}^{-1}\right)$, so that increased intensity of precipitation in the future climate implies that the threshold is exceeded more regularly, and hence events are longer. The sensitivity of our analysis to the minimum threshold is investigated in the appendix. 

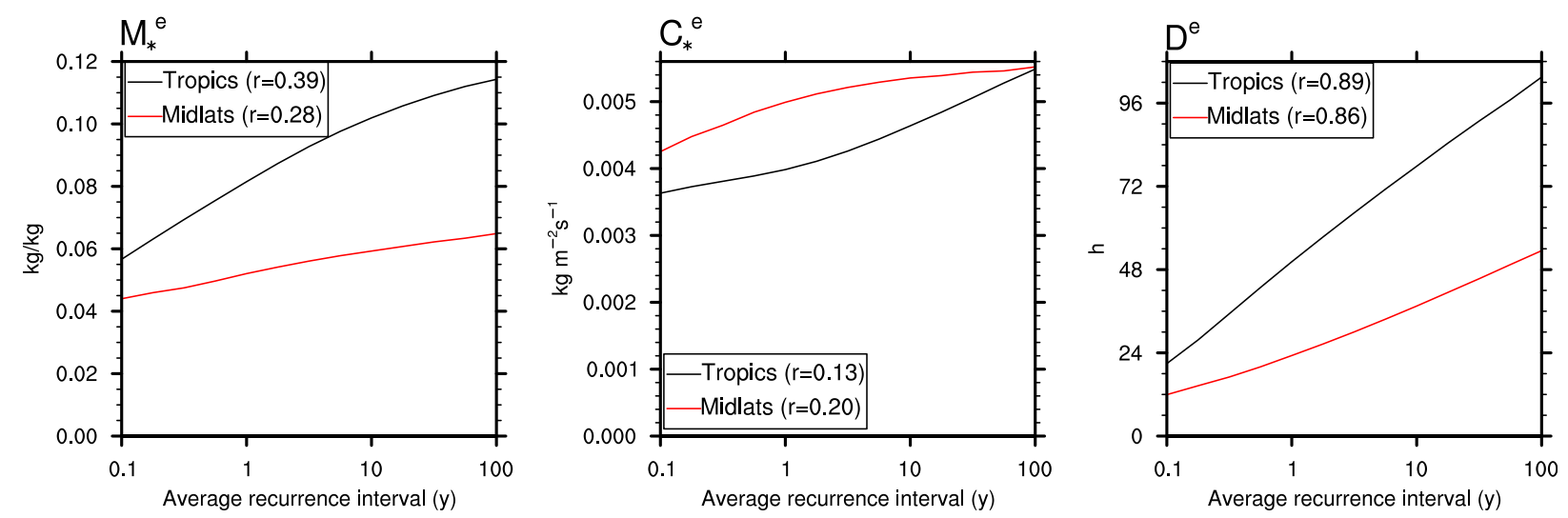

FIG. 3. Terms $M_{*}, C_{*}$, and $D$, representing moisture, circulation, and duration of events, shown as a function of ARI of accumulated precipitation in E20. The curves are calculated at each grid point by conditionally averaging at each ARI (section 3c), then the curves are averaged over all land grid points between $15^{\circ} \mathrm{S}$ and $15^{\circ} \mathrm{N}$ (black) and between $40^{\circ}$ and $50^{\circ}$ in each hemisphere (red). The given $r$ values are the correlations of the given variable with $P_{\text {acc }}$ from all events registering as accumulations (section $2 \mathrm{~b}$ ): the correlations are calculated at each grid point, and then these correlations are averaged over the same grid points as for the curves.

\section{c. $M_{*}, C_{*}$, and $D$ as a function of $A R I$ of $P_{a c c}$}

In this study, we conditionally average $M_{*}, C_{*}$, and $D$ for each of the ARIs of $P_{\text {acc }}$ shown in Table 1 to obtain

$$
P_{\mathrm{acc}}^{e} \approx M_{*}^{e} C_{*}^{e} D^{e}
$$

where $X^{e}$ denotes the conditional mean of some quantity $X$ at the $e_{i}$-yr ARI of $P_{\text {acc }}$, obtained by averaging $X$ across all events between the $e_{i-1 / 2}$-yr and $e_{i+1 / 2}$-yr ARIs (see section 2c). In addition to the neglected terms previously noted, this approximation neglects the covariance between $C_{*}, M_{*}$, and $D$ across events for a given ARI.

The values of $M_{*}^{e}, C_{*}^{e}$, and $D^{e}$ as a function of ARI of $P_{\text {acc }}$, averaged over the tropics and midlatitudes, are shown in Fig. 3, along with the mean correlations of each variable with $P_{\mathrm{acc}}$. All three factors are monotonic increasing with ARI and positively correlated with $P_{\text {acc }}$, indicating that large values of all three factors are generally associated with large accumulations. However, $M_{*}$ is weakly correlated with $P_{\text {acc }}(r=0.39$ in the tropics and 0.28 in the midlatitudes) and $C_{*}$ is more weakly correlated with $P_{\text {acc }}(r=0.13$ in the tropics and 0.20 in the midlatitudes). Hence, large values of moisture or convergence do not necessitate a large accumulation. Note that for both moisture and convergence, these are the mean values during an event and would likely be more highly correlated with precipitation rate during the events than with accumulation. By contrast, $D$ is highly correlated with $P_{\text {acc }}(r=0.89$ in the tropics and 0.86 in the midlatitudes), strongly indicating that an event that is longer lasting tends to produce a large accumulation. There is also a greater fractional increase of $D$ from low to high ARIs than for $M_{*}$ or $C_{*}$, increasing from $22 \mathrm{~h}$ for the $0.1-\mathrm{yr}$ accumulation to $103 \mathrm{~h}$ for the $100-\mathrm{yr}$ accumulation in the tropics, and from $12 \mathrm{~h}$ for the $0.1-\mathrm{yr}$ accumulation to $53 \mathrm{~h}$ for the $100-\mathrm{yr}$ accumulation in the midlatitudes.

\section{d. Calculating the risk ratio for a given accumulation size}

Given the curves of accumulated precipitation as a function of ARI in E20 and E21 separately, the change in frequency of a given accumulation size from E20 to E21 may be calculated. This technique is demonstrated in Fig. 4a in which hypothetical curves for E20 and E21 are plotted. In this example, accumulation increases logarithmically from the 0.1- to 100-yr ARI, indicating that all of the accumulation sizes represented on this scale are above the cutoff (Neelin et al. 2017), defined in the introduction, that is, the frequency of exceedance decreases exponentially with accumulation size. To obtain the E21 curve, the precipitation accumulations are assumed to scale with warming: specifically, at each ARI the E21 accumulation is obtained by multiplying the E20 accumulation by 1.2 , that is, by $7 \% \mathrm{~K}^{-1}$ warming, hence by $20 \%$ for the approximately $3-\mathrm{K}$ global-mean surface warming from E20 to E21. The ARI in E21 for the accumulation size corresponding to some ARI in E20 is calculated by linearly interpolating on the E21 curve to find the ARI at which the same accumulation size is exceeded in E21. Although the accumulation at each ARI is enhanced by the same proportional amount, the 1-, 10-, and 100-yr accumulations in E20 become the $0.48-, 3.2-$, and 22-yr accumulations, respectively.

We define the risk ratio for the $i$ th $\mathrm{ARI}$ as $e_{i, \mathrm{E} 20} / e_{i, \mathrm{E} 21}$, where $e_{i, \mathrm{E} 20}$ is the $i$ th ARI in E20, corresponding to some accumulation size, and $e_{i, \mathrm{E} 21}$ is the interpolated ARI on the 
(a) Accumulation size

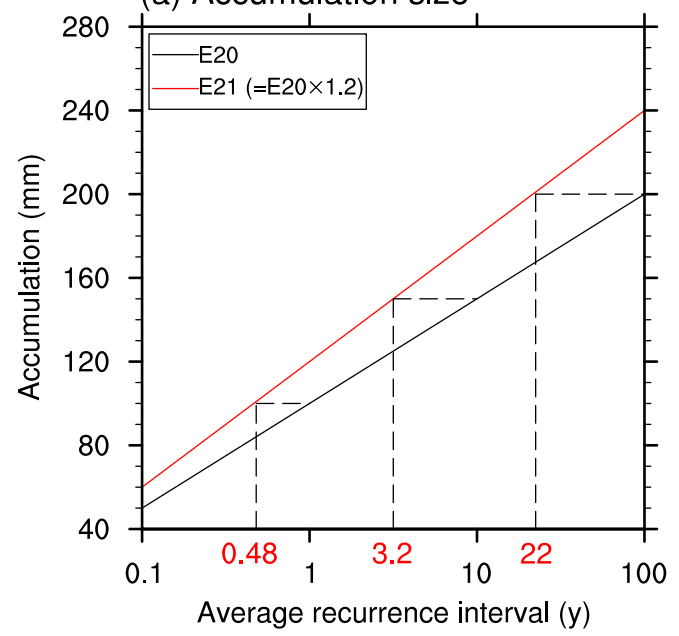

(b) Risk ratio

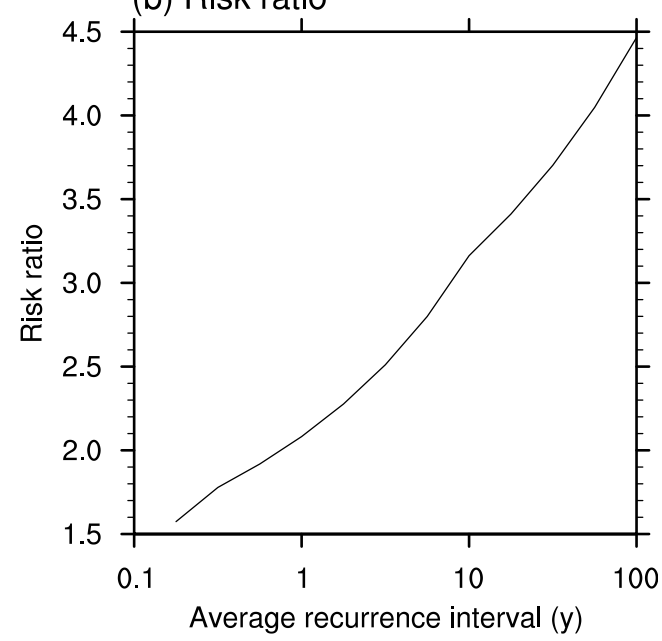

FIG. 4. (a) A hypothetical example showing the accumulation size corresponding to each ARI, both in the current (E20) and projected future (E21) climates. The E21 accumulations are calculated by multiplying the E20 accumulation at each ARI by 1.2 , according to a $20 \%$ increase in moisture content, which is expected from a $3-\mathrm{K}$ warming. For each ARI in E20, the corresponding ARI in E21 is found at which the same accumulation size is exceeded. For the 1-, 10- and 100-yr accumulations during E20, the associated ARI during E21 is indicated in red on the $x$ axis. (b) The risk ratio for the same example, obtained by dividing the ARI in E20 by the ARI in E21. For example, the 1-, 10-, and 100-yr accumulations in E20 are the 0.48-, 3.2-, and 22-yr accumulations in E21, making the risk ratio 2.1,3.1, and 4.5, respectively. If a given accumulation size does not lie on the E21 curve (i.e., because its ARI is $<0.1$ years), then the risk ratio is undefined.

E21 curve, representing the number of years in E21 between exceedances of the same accumulation size. Hence, in the example in Fig. 4a, the risk ratios for the 1-, 10-, and 100-yr accumulations are 2.1, 3.1, and 4.5, respectively (Fig. 4b). This means that in the future, the 1-yr accumulation becomes about twice as frequent, the 10-yr accumulation becomes about 3 times as frequent, and the $100-\mathrm{yr}$ accumulation becomes about 5 times as frequent. This example illustrates the effect of simply increasing moisture content on the accumulation cutoff, so that the risk ratio is greatest for the most extreme events, similarly to the results of Chou et al. (2012, their Fig. 5a) in the tropics.

In reality, changes to moisture, convergence, and duration, represented by $M_{*}, C_{*}$, and $D$, respectively, will each impact the magnitude of accumulation at each ARI in the future climate, as will be shown, so that we may identify the risk ratio as a function of ARI (Fig. 4b) for each individual factor, that is, how much would the frequency change if only moisture, or only convergence, or only duration of events were to change? Note that risk ratio is a nonlinear process, so that the sum of risk ratios due to individual factors does not equal the actual risk ratio. For example, a risk ratio of 2 associated with each of the three factors does not imply a total risk ratio of 6 .

We acknowledge that the frequency of a given magnitude may undergo a large increase without a great increase in magnitude for a given frequency. For example, if the $100-y r$ accumulation is only slightly greater than the 10 -yr accumulation, then just a slight future increase in the 10-yr event could lead to a tenfold increase in frequency of that accumulation size. Therefore, plotting the curve of accumulation size versus ARI is useful for confirming that large increases in frequency are meaningful, as will be done for our data.

Note that risk ratio cannot be calculated where a given accumulation size does not lie on the E21 curve. In Fig. 4, this is the case for the 0.1-yr accumulation because the 0.1-yr accumulation in E21 is greater than in E20 and this is the lowest ARI. Therefore, the lowest ARIs are included in the analysis mainly just to calculate risk ratio for higher ARIs. Similarly, the risk ratio for the 100 -yr accumulation cannot be calculated if the 100-yr accumulation in E21 is less than in E20 (because this is the highest ARI). Where this is the case, a risk ratio $<1$ indicates decreasing frequency, which is shown in various figures, but in these situations the precise risk ratio has not been calculated. Because the 100-yr accumulation almost universally increases, this issue impacts the results minimally.

\section{Evaluation of simulated accumulations compared to observations}

Before analyzing projected changes to accumulations, an evaluation of model skill is first conducted for the 
(a) Tropics

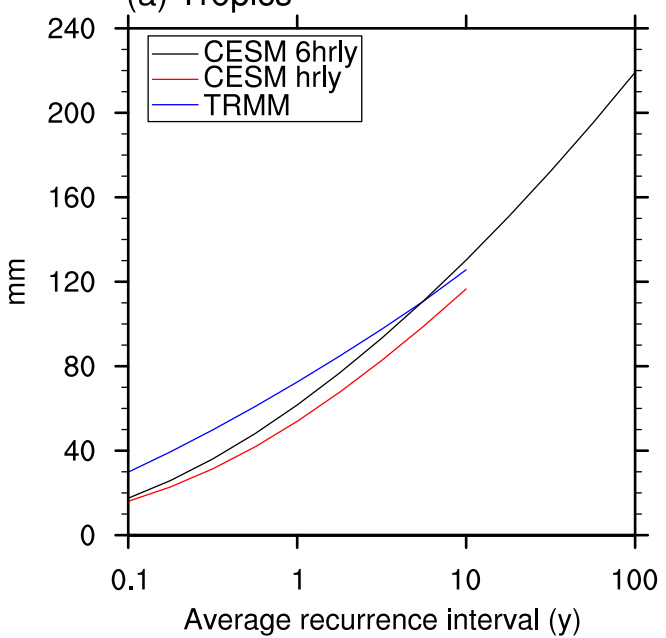

(b) Midlatitudes

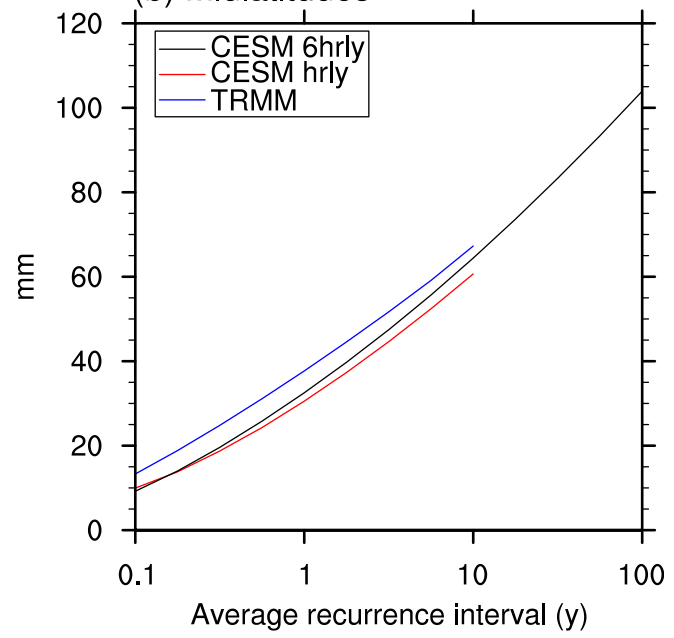

FIG. 5. The accumulation size corresponding to each ARI in E20, comparing 6-hourly CESM, hourly CESM, and 3-hourly TRMM 3B42. The curves are obtained by averaging at each ARI across all land grid points (a) between $15^{\circ} \mathrm{S}$ and $15^{\circ} \mathrm{N}$ and (b) between $40^{\circ}$ and $50^{\circ}$ in each hemisphere. Due to a smaller amount of data, the CESM hourly and TRMM curves are only shown up to the $10-\mathrm{yr}$ accumulation.

current climate. The first important evaluation to be made is whether 6-hourly output is sufficient for accurately representing accumulations. During a 6-h period, precipitation may stop and restart, so that two consecutive 6-hourly time slices may sample two separate accumulations, but mistakenly take them to be the continuation of a single accumulation. This effect is likely the cause of the slight overestimate of 6-hourly compared to hourly CESM accumulations (Fig. 5: compare black and red curves). For the 0.1-, 1-, and 10 -yr accumulations, the mean accumulation over tropical land is $9 \%, 14 \%$, and $12 \%$ greater for 6-hourly than hourly data (Fig. 5a), but the discrepancies are lower in the midlatitudes $(7 \%$ less, $6 \%$ greater, and $6 \%$ greater for the 0.1-, 1-, and 10-yr accumulations; Fig. 5b). The tropics are the most sensitive to output time perhaps because deep convective events may stop and start on an hourly time scale. By contrast, higher-latitude events, for example, extratropical cyclones, occur more on a daily time scale (i.e., consecutive fronts are usually at least a day apart), so that there is little discernable difference between hourly and 6-hourly data when averaged over a large number of events.

Greater discrepancies exist between CESM and TRMM, although these reduce proportionally with increasing ARI (Fig. 5: compare black and blue curves). In the tropics, for the 0.1-, 1-, and 10-yr accumulations, the CESM accumulation is $59 \%, 85 \%$, and $104 \%$ of the TRMM accumulation (Fig. 5a). Evidently, this underestimate is not due to insufficient temporal resolution because the hourly CESM data show even lower accumulations. The underestimate is most likely because of the convective parameterization in climate models that fails to represent convective updrafts (e.g., O'Gorman and Schneider 2009; Berg et al. 2013; Kharin et al. 2013). The closer agreement between CESM and TRMM for larger ARIs may be because averaging over longer events yields less error. There is generally closer agreement in the midlatitudes (for the 0.1-, 1-, and 10-yr accumulations the CESM accumulation is $69 \%, 86 \%$, and $96 \%$ of the TRMM accumulation; Fig. 5b), likely because climate models are more reliable at simulating the magnitude of frontal than convective ascent (O'Gorman and Schneider 2009; Berg et al. 2013; Sillmann et al. 2013).

For the tropics and midlatitudes, there is agreement between CESM and TRMM in the relationship between accumulation size and ARI: in particular, the accumulation increases approximately logarithmically with ARI, approximately doubling for each order of magnitude increase in ARI. This feature indicates that the range of accumulations represented by our methodology is almost all above the cutoff, defined in Neelin et al. (2017), that is, an exponential decrease in frequency with increasing accumulation size, in agreement with the conceptual example in Fig. 4. Agreement in this regard is important because the shape of the curve in the current climate determines the future risk ratio (Fig. 4). Also, because TRMM is only used for evaluation of CESM for ARIs up to 10 years, due to TRMM's limited data period, the agreement in the shape of the curve lends confidence to the magnitudes of $100-\mathrm{yr}$ events in 

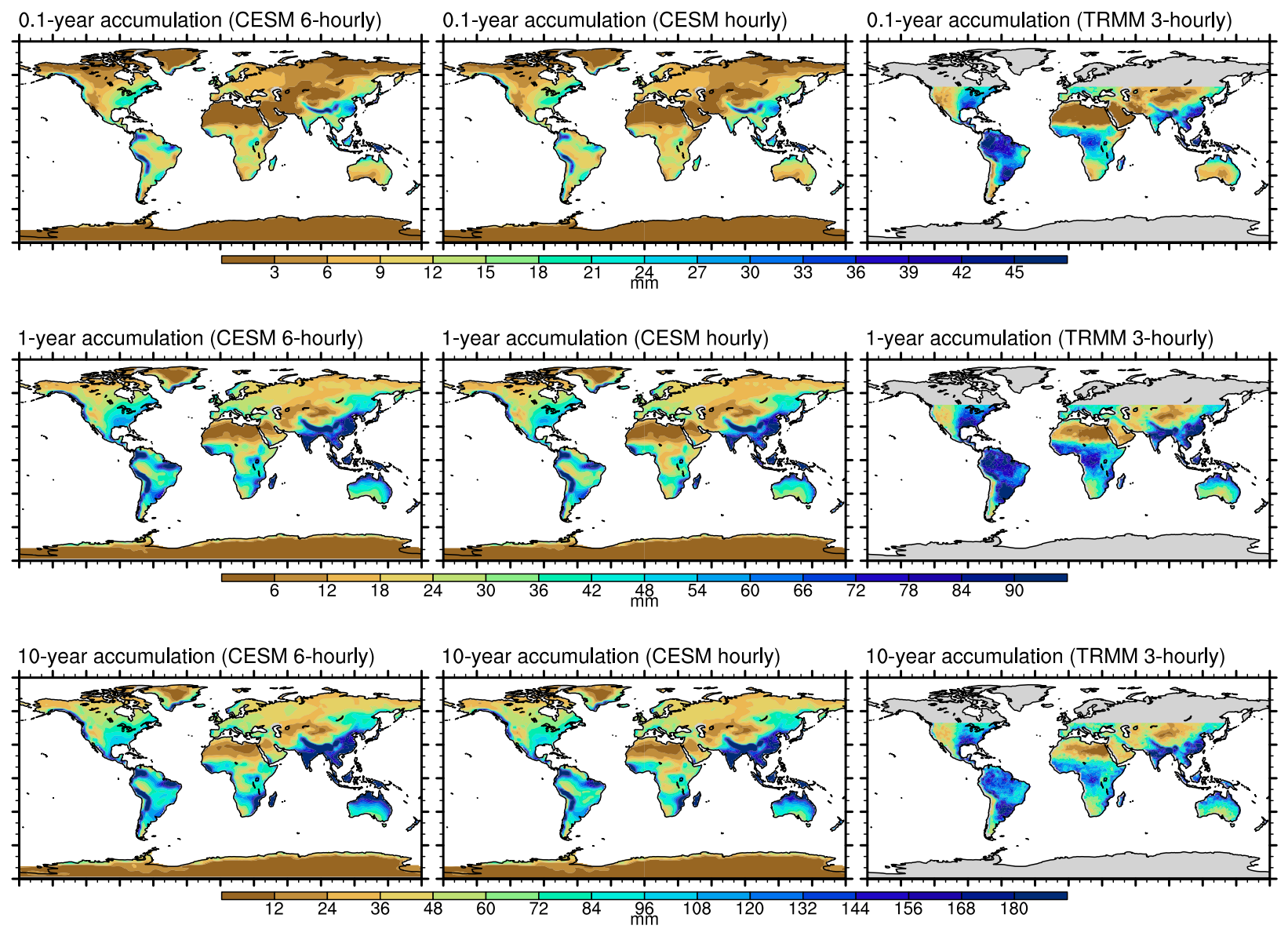

FIG. 6. The accumulation size whose ARI is (top) 0.1 , (middle) 1 , and (bottom) 10 years, comparing (left) 6-hourly CESM, (center) hourly CESM, and (right) TRMM 3B42. Regions where TRMM data are missing are colored gray.

CESM. We note that ARIs above 10 years could be evaluated at individual locations where multidecadal rain gauge data exist, which would be appropriate for regional evaluation of simulated accumulations. However, the focus of this study is on the global patterns.

Inspection of the spatial maps of accumulation for different ARIs, comparing between 6-hourly CESM, hourly CESM, and TRMM, reveal that accumulations in some regions are more accurately simulated than in others (Fig. 6). The spatial patterns of 6-hourly and hourly CESM data are almost identical at each ARI (cf. left and center columns) with Pearson correlation coefficients $r$ over land of 1.00 for all of the ARIs shown. Comparing CESM and TRMM (left and right columns), the large underestimate of CESM in the tropics for short ARIs (0.1 years) is readily evident, and, although there is closer agreement at greater ARIs, it is clear that some regions are better simulated than others. In particular, the Amazon, the Congo basin, and the eastern United States are massively underestimated for all ARIs. But, for the 1- and 10-yr accumulations, there is good agreement for the tropical parts of southern and eastern Asia. And, for all ARIs there is good agreement in southern Africa, southern South America, the western United States, Australia, and central Asia.

The dry biases of climate models in particular regions have been documented in the literature. For example, over the Amazon, climate models have a dry bias due to an underestimate of moisture convergence, which may result from their inability to represent SST variability in the equatorial Pacific and Atlantic (Martins et al. 2015). Over the Congo, there is a large intermodel spread in precipitation between CMIP5 models, where drier models typically feature a more equatorward African easterly jet (Creese and Washington 2018). This feature disrupts the overturning circulation over the eastern tropical Atlantic, suppressing rainfall over the Congo. Over the eastern United States, the underestimate is likely explained by the relevant events in this region being in the cool season, according to CESM, but in the warm season, according to TRMM (Fig. S1 in the online supplemental material-note that CESM and TRMM 
agree that there is no discernable difference in seasonality between different ARIs). This likely arises from the poor representation of tropical cyclones in climate models (Camargo and Wing 2016), so that CESM erroneously identifies winter events as the largest accumulations. Over these regions where the comparisons are poorer, the future projections are less reliable.

With the caveat of poor model performance in certain regions, we note that globally the spatial patterns between CESM and TRMM are reasonable $(r=0.67,0.74$, and 0.75 for the 0.1-, 1-, and 10-yr accumulations over land in the area covered by TRMM) and that globally the magnitudes are reasonable, particularly for greater ARIs (for the 0.1-, 1-, and 10-yr accumulations the mean CESM accumulation is $65 \%, 93 \%$, and $113 \%$ of the mean TRMM accumulation). These comparisons with TRMM and hourly CESM data lend confidence to the projected changes to the frequency of large accumulations over most of the global land analyzed in the remainder of this paper.

\section{Projected changes to the frequency of large accumulations}

Figure 7 shows the risk ratios of the accumulation sizes whose ARIs in the current climate are 1, 10, and 100 years (left panels). For all accumulation sizes, the majority of global land experiences enhanced frequency, consistent with other studies projecting that large precipitation totals or accumulations will become more frequent (e.g., Kao and Ganguly 2011; Chou et al. 2012; Kharin et al. 2013; Dwyer and O'Gorman 2017; Neelin et al. 2017). For the 1-yr accumulation, there is a relatively small increase in frequency, with almost all locations experiencing a risk ratio of $<2$, that is, less than a doubling of frequency. However, with increasing ARI, the risk ratio becomes increasingly large and the locations where there are projected decreases in frequency (the Amazon and small areas in the subtropics) become fewer. For the 100-yr accumulation, almost the entire globe experiences a risk ratio of $>2$, and large areas, mostly in the tropics and monsoon regions, experience a risk ratio of $>5$. The spatial patterns for the 10- and 100-yr accumulations are similar to the fractional increase of the $10-\mathrm{yr}$ maximum of daily precipitation, also using the CESM Large Ensemble (Tandon et al. 2018), indicating that accumulations and fixed-period totals are subject to the greatest enhancements in similar areas. Given that the 100-yr accumulation is nearly double the 20 -yr accumulation, averaged over the tropics (Fig. 5a), a 5 time increase in frequency of the 100-yr accumulation (i.e., the $100-\mathrm{yr}$ event becomes the 20 -yr event) represents a major increase to flood risk.
In Eq. (7), this approximation was made: $P_{\mathrm{acc}}^{e} \approx$ $M_{*}^{e} C_{*}^{e} D^{e}$. That is, an accumulation is the product of three 2D variables representing moisture, low-level convergence, and duration, all conditionally averaged at the given ARI of $P_{\text {acc }}$. Equation (7) is evaluated in Fig. S7 and discussed in the appendix. However, more relevantly for this study, the risk ratios for various accumulation sizes (Fig. 7, left panels) are almost the same as the risk ratios if only $M_{*}^{e}, C_{*}^{e}$, and $D^{e}$ are allowed to change from the present to future climate (right panels). That is, the right panels show the risk ratio of $P_{\text {acc }}$ obtained by making the approximation

$$
\Delta P_{\mathrm{acc}}^{e} \approx \Delta\left(M_{*}^{e} C_{*}^{e} D^{e}\right),
$$

where $\Delta X^{e}$ denotes the change of some quantity $X$ from E20 to E21 at the $e$ th ARI of $P_{\text {acc. }}$. The risk ratio based on $\Delta\left(M_{*}^{e} C_{*}^{e} D^{e}\right)$ is in most locations marginally greater than the risk ratio based on $\Delta P_{\text {acc }}^{e}$ (cf. left and right panels), due to a negative influence of the horizontal advection term, $-\Delta\left(D^{e} \overline{\{\mathbf{v} \cdot \nabla q\}^{e}}\right)$ (Fig. S10), which is neglected in (8). Therefore, we proceed to decompose the changes to accumulations into the contributions of moisture, convergence, and duration, given that the risk ratio due to these three factors collectively is approximately the same as the actual risk ratio of the accumulations.

\section{Changes to magnitude of accumulations due to $M_{*}, C_{*}$, and $D$}

In this section, we address how much larger the accumulations of a given ARI are projected to become due to $M_{*}, C_{*}$, and $D$ individually. This is the first step in calculating how much more frequent given accumulation sizes will become due to each factor. For example, if the 100-yr accumulation in the future is greater than the 100-yr accumulation in the present climate, then the present-climate 100-yr accumulation must be more frequently exceeded in the future.

To address this issue, future-climate projections of $P_{\mathrm{acc}}^{e}$ are calculated by adding the change to just one factor on the right-hand side of (7). In particular, we calculate, for the $e$-yr ARI

$$
\begin{gathered}
\text { (i) } C_{*, \mathrm{E} 20}{ }^{e} D_{\mathrm{E} 20}^{e} \Delta M_{*}^{e}, \quad \text { (ii) } M_{*, \mathrm{E} 20}{ }^{e} D_{\mathrm{E} 20}^{e} \Delta C_{*}^{e}, \\
\text { (iii) } M_{*, \mathrm{E} 20}{ }^{e} C_{*, \mathrm{E} 20}^{e} \Delta D^{e},
\end{gathered}
$$

where $\Delta X^{e}=X_{\mathrm{E} 21}^{e}-X_{\mathrm{E} 20}^{e}$ denotes the change of some quantity $X$ at the $e$-yr ARI from E20 to E21, and $X_{\mathrm{E} 20}^{e}$ and $X_{\mathrm{E} 21}^{e}$ denote some quantity $X$ conditionally averaged at the $e$-yr ARI in E20 and E21, respectively. 

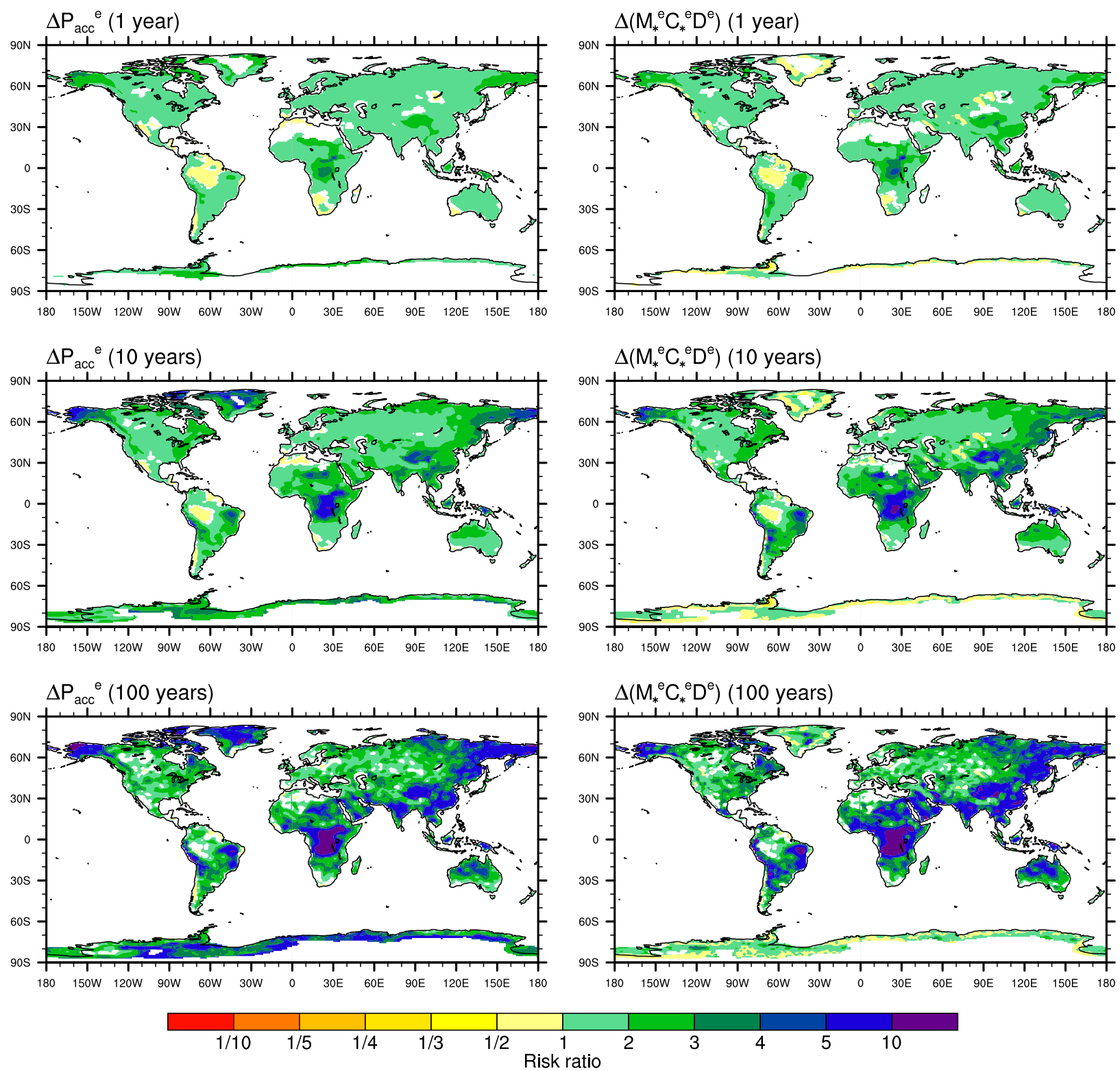

FIG. 7. (left) The risk ratio of the accumulation size whose ARI in E20 is (top) 1, (middle) 10, and (bottom) 100 years, calculated by comparing the curve of ARI vs accumulation size between E20 and E21 (such as in Fig. 4) at each grid point. Wet and dry colors indicate an increased and decreased frequency, respectively. Values are only plotted where $>80 \%$ of bootstrap replications agree on whether the $1-$, $10-$, or 100-yr accumulation increases or decreases. (right) As in the left panels, but calculated by comparing the curves of $P_{\text {acc }}^{e}$ in E20 and $P_{\mathrm{acc}}^{e}+\Delta\left(M_{*}^{e} C_{*}^{e} D^{e}\right)$, i.e., the risk ratio if just those three factors are allowed to change, representing moisture, circulation, and duration of events, respectively.

Similarly to the hypothetical curves in Fig. 4a, Fig. 8 shows $P_{\text {acc }}$ as a function of ARI, averaged over the tropics (top row) and midlatitudes (bottom row), comparing the current climate (black curves) and that with the expressions in (9) added at each ARI (red curves) to establish the increase in accumulation size if only moisture (second column), convergence (third column), or duration (fourth column) were to change. Also shown is the actual increase in accumulation size (first column), which is approximately the sum of the increases shown in the other panels.

Of the three factors, moisture plays the most important role in increasing accumulation size by about $20 \%$ at each ARI (Fig. 8, second column), as expected from ClausiusClapeyron for the 3.3-K warming from E20 to E21. In both the tropics and midlatitudes, this is approximately 

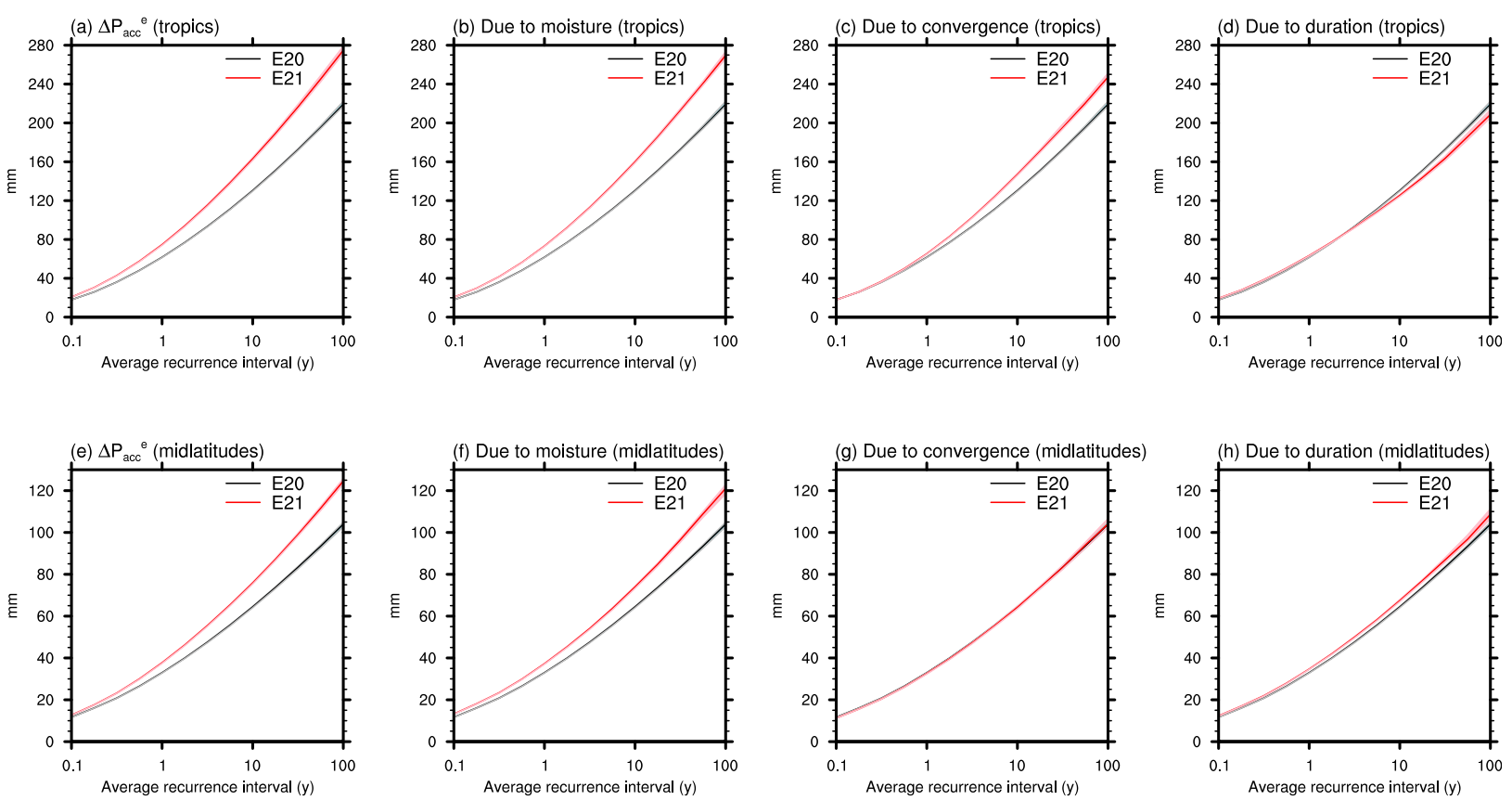

FIG. 8. (first column) Accumulation size as a function of ARI in E20 (black) and E21 (red). The other panels compare accumulation size in E20 $P_{\mathrm{acc}}^{e}$ with the accumulation size (second column) if only moisture changes $\left(P_{\mathrm{acc}}^{e}+C_{*}^{e} D^{e} \Delta M_{*}^{e}\right)$, (third column) if only convergence changes $\left(P_{\mathrm{acc}}^{e}+M_{*}^{e} D^{e} \Delta C_{*}^{e}\right)$, and (fourth column) if only duration changes $\left(P_{\mathrm{acc}}^{e}+M_{*}^{e} C_{*}^{e} \Delta D^{e}\right)$, where $X^{e}$ represents $X$ at the $e$-yr ARI in E20 and $\Delta X^{e}$ represents the change of $X$ from E20 to E21 at the $e$-yr ARI. The curves are averaged for the given ARI over all land grid points (top) between $15^{\circ} \mathrm{S}$ and $15^{\circ} \mathrm{N}$ and (bottom) between $40^{\circ}$ and $50^{\circ}$ in each hemisphere. The full spread of bootstrap replications is shown for both curves by the shaded areas, hence wherever the gray and red shaded areas do not overlap, there is a significant change from E20 to E21.

equal to the actual increase in accumulation size (left panels). Because this is the event-mean moisture, this figure illustrates an increase in intensity of precipitation for a given ARI of accumulation, consistent with previous studies investigating future changes to precipitation extremes (Pfahl et al. 2017; Tandon et al. 2018; Norris et al. 2019). The dominance of moisture over other factors is shown at individual locations in Fig. 9 (changes due to moisture are shown in left panels), with the exception of parts of the tropics, particularly at higher ARIs.

In the tropics, convergence accounts for a statistically significant increase in accumulation size for ARIs above about 1 year (Fig. 8c). However, except for the most extreme accumulations, the increase in accumulation size is small. With increasing ARI, more of the tropics shows a significant increase due to convergence (Fig. 9, middle column) and the 100-yr accumulation is increased by about $15 \%$ due to convergence (Fig. $8 \mathrm{c}$ ), similarly to the effect of moisture. However, this is partly offset by a decrease in duration for the 100 -yr accumulation by about $10 \%$ (Fig. 8d), explaining why the increase due to moisture is similar to the actual increase in accumulation size (Figs. 8a,b). The spatial distributions of the changes due to convergence and duration illustrate that in the tropics the positive effect of convergence is not necessarily collocated with the negative effect of duration. In particular, South and East Asia and northern Australia show a large increase due to convergence, but only a slight compensating decrease due to duration (Fig. 9, cf. middle and right columns). Therefore, at high ARIs in these locations, the increase of accumulation size is far greater than due to moisture alone.

Averaged over the midlatitudes, by contrast, there are no significant enhancements of events of any accumulation size due to convergence (Fig. 8g). The spatial patterns reveal that for the $1-y r$ accumulation, there are decreases due to convergence in some subtropical and midlatitude locations (Fig. 9, middle column), similarly to the dynamical component of projected precipitation extremes (Pfahl et al. 2017; Tandon et al. 2018; Norris et al. 2019), which are related to an increasing horizontal scale of vertical velocity anomalies (Tandon et al. 2018). However, with increasing ARI, a smaller part of the globe exhibits significant decreases due to convergence.

By contrast, in the midlatitudes, there are small but significant increases in accumulations associated with duration at all ARIs (Fig. 8h), resulting from an increase in duration at each ARI. The increase associated with 

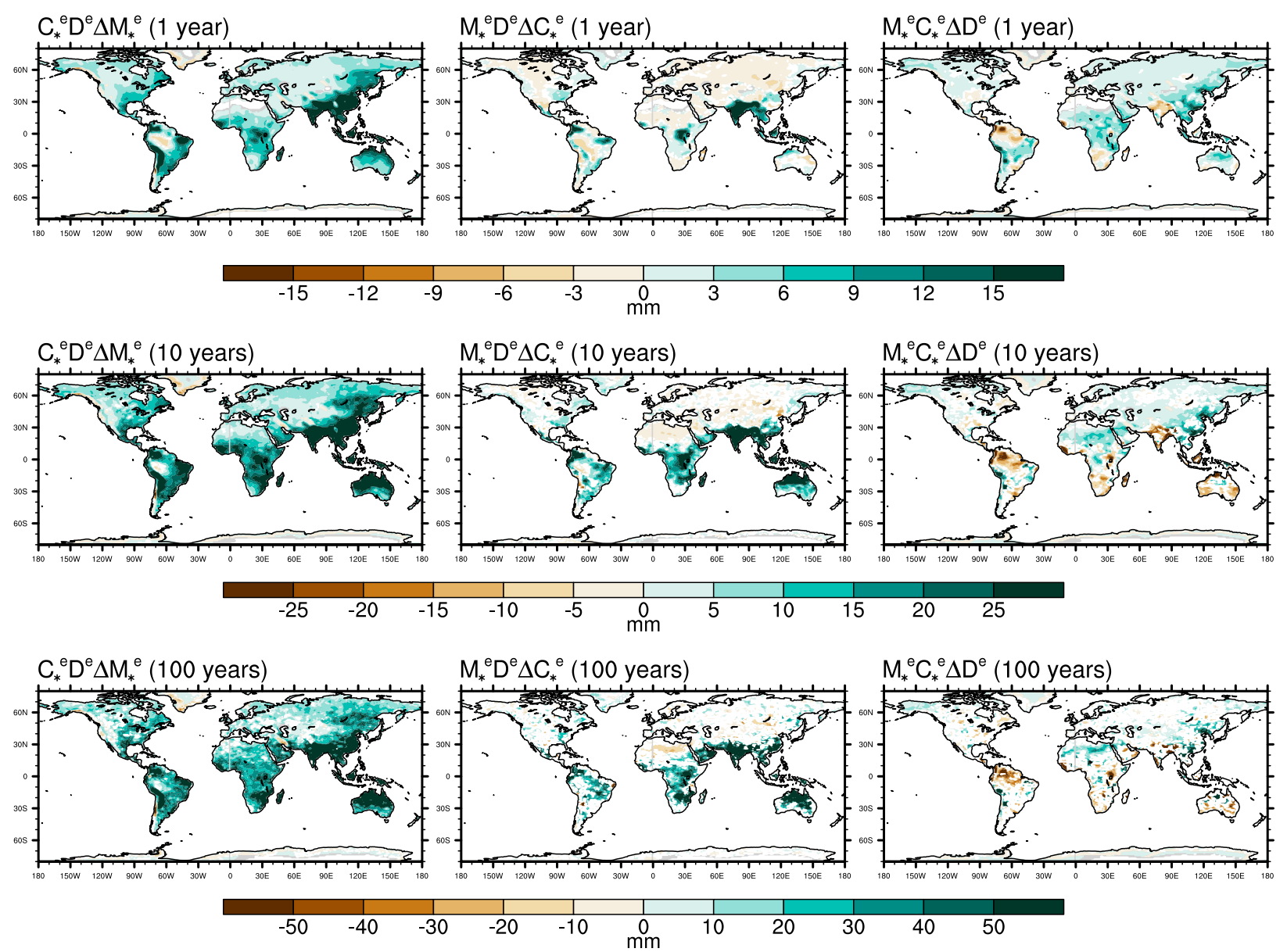

FIG. 9. The future changes to the (top) 1-, (middle) 10-, and (bottom) 100-yr accumulations due to (left) moisture, (center) convergence, and (right) duration. In the given expressions, $X^{e}$ represents $X$ at the $e$-yr ARI in E20 and $\Delta X^{e}$ represents the change of $X$ from E20 to E21 at the $e$-yr ARI. Values are only plotted where $>80 \%$ of bootstrap replications agree on whether the given factor increases or decreases for the 1-, 10-, or 100-yr accumulation.

duration is widespread over the midlatitudes for the 1and 10-yr accumulations, but noisy for the 100-yr accumulation (Fig. 9, right column). By contrast Dwyer and O'Gorman (2017) showed that the duration of extreme events in the midlatitudes is projected to decrease by about $1 \% \mathrm{~K}^{-1}$ warming. However, they showed that the duration of events with high precipitation rate will decrease, whereas we show that the duration of events delivering large accumulations will increase. Our results are consistent with Martinez-Villalobos and Neelin (2018), who identified events by accumulation size, based on precipitation observations over North America since 1979, and found increases in duration. Although these are not major increases due to duration, they indicate that large accumulations are projected to increase by slightly more than due to moisture alone (cf. Figs. 8e,f). Note that the projection of changing duration of events is not greatly sensitive to the minimum precipitation rate used to calculate accumulations, and the results described above are also valid if using a threshold of 0.1 , rather than $0.5 \mathrm{~mm} \mathrm{~h}^{-1}$ (Fig. S6).

We acknowledge that these projections are based on the thermodynamic $\left(\Delta M_{*}^{e}\right)$ and dynamic $\left(\Delta C_{*}^{e}\right)$ effects from just one model and, although moisture increases are robust among climate models, the dynamical component of extreme events in the tropics varies between climate models (O'Gorman and Schneider 2009). The results are therefore presented with this caveat, but also acknowledging that models are in agreement that there is a dynamical enhancement of extreme events in the tropics (Pfahl et al. 2017), with disagreement only in the magnitude thereof.

\section{Risk ratio due to $M_{*}, C_{*}$, and $D$}

In this section, we take the changes to accumulation size due to moisture, convergence, and duration, depicted 
(a) Tropics

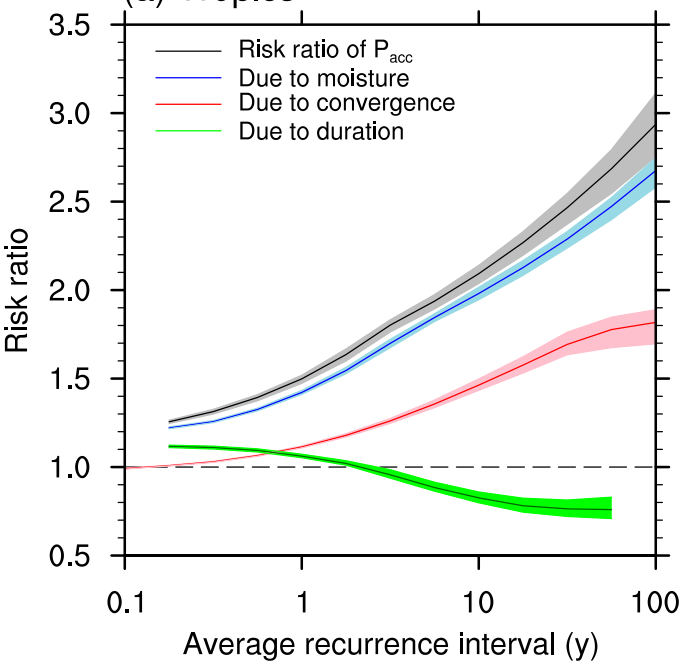

(b) Midlatitudes

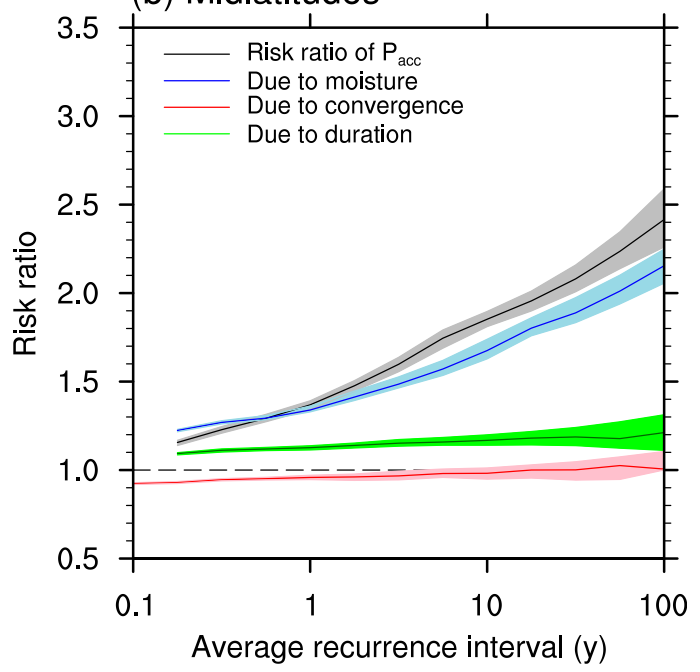

FIG. 10. The risk ratio of the accumulation size corresponding to each ARI in E20 (black curves). Risk ratio is calculated as the given ARI divided by the ARI of the given accumulation size in E21, as conceptualized in Fig. 4b. The other curves in each panel show the risk ratio if only moisture (blue), mass convergence (red), or duration of events (green) were to change, calculated by comparing the black and red curves in Fig. 8 . The curves are averaged for the given ARI over all land grid points (a) between $15^{\circ} \mathrm{S}$ and $15^{\circ} \mathrm{N}$ and (b) between $40^{\circ}$ and $50^{\circ}$ in each hemisphere. The dashed line in each curve is where the risk ratio is 1, i.e., where a curve is above the line, events become more frequent, and below the line events become less frequent. If a given accumulation size does not lie on the E21 curve in Fig. 8 (i.e., because it is smaller than the 0.1-yr accumulation or greater than the 100-yr accumulation), then the risk ratio is undefined, e.g., for the 0.1-yr accumulation for most factors, and for the 100-yr accumulation for duration in the tropics (see section 3d). The shaded areas show the full spread of bootstrap replications for the given curve, indicating the robustness of the risk ratio of each factor.

in Figs. 8 and 9, to answer the question, how much of the projected frequency increase of large accumulations (Fig. 7) is due to each of these factors? This question is addressed in Fig. 10, which shows the risk ratio of $P_{\text {acc }}^{e}$ (black curves) and the risk ratio due to the individual factors (colored curves). The risk ratio is calculated by comparing the E20 and E21 curves in Fig. 8, as conceptualized in Fig. 4. Note that the summation of colored curves does not necessarily equal the actual risk ratio (black) because increasing frequency is a nonlinear process.

As previously illustrated in Fig. 7, the risk ratio of precipitation accumulation increases with ARI, with the 100-yr event becoming 2.9 times as frequent averaged over the tropics and 2.4 times in the midlatitudes (Fig. 10, black curves). In agreement with the conceptual example in Fig. 4, this results from the increase in the cutoff of large accumulations (Neelin et al. 2017)accumulation sizes that in the current climate are the farthest beyond the cutoff are subject to the greatest increase in frequency as the cutoff increases.

For all latitudes, the risk ratio due to enhanced moisture (about $20 \%$ in the warmer climate; Fig. 8 ) increases approximately logarithmically with ARI to 2.7 for the $100-y r$ return in the tropics and 2.2 in the midlatitudes (Fig. 10, blue curves), meaning that moisture alone makes the 100 -yr accumulation more than twice as frequent. This result is consistent with Chou et al. (2012), who used climate models to predict about a $40 \%$ increase in frequency of the most extreme daily rainfall totals in the tropics per degree of warming (hence, slightly more than a doubling of frequency for a 3-K warming). Our results indicate that this also applies outside the tropics. Analysis at individual locations shows that the risk ratio due to moisture is relatively spatially uniform compared to other factors (Fig. 11, moisture shown on top row), with most of the globe exhibiting a risk ratio due to moisture of $<2$ for the 10 -yr accumulation and 2-3 for the 100-yr accumulation.

In the tropics, the risk ratio due to moisture is slightly less than the actual risk ratio of $P_{\text {acc }}$ (Fig. 10a, compare black and blue curves). At low ARIs ( $<1$ year), the discrepancy is due to increasing duration, with convergence having no impact. However, with increasing ARI, convergence has a greater impact, responsible by itself for almost a doubling of frequency for the 100 -yr accumulation. However, the negative effect of duration partially cancels the positive effect of convergence, so that the actual risk ratio of $P_{\text {acc }}$ for the 100 -yr accumulation is only slightly more than due to moisture alone. 
10-year accumulation: if only moisture changes

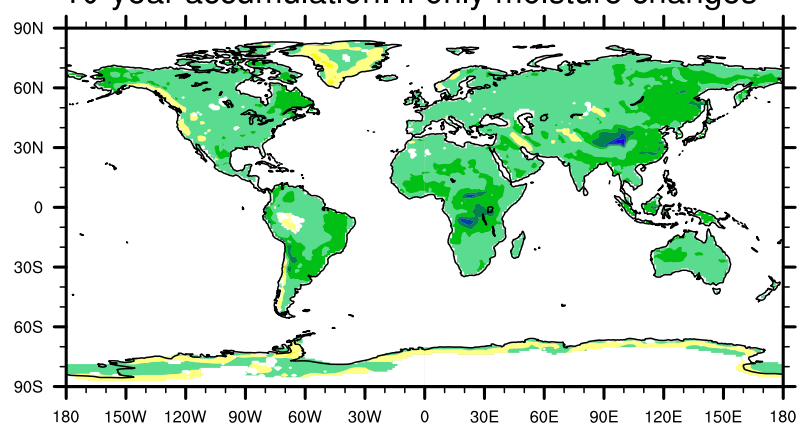

10-year accumulation: if only circulation changes

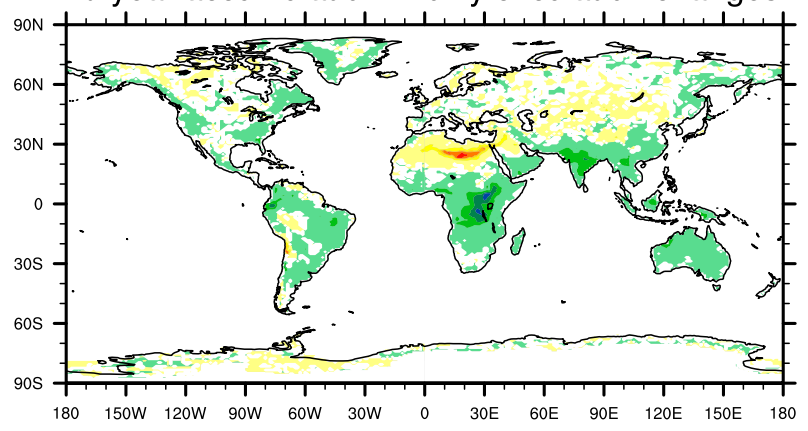

10-year accumulation: if only duration changes

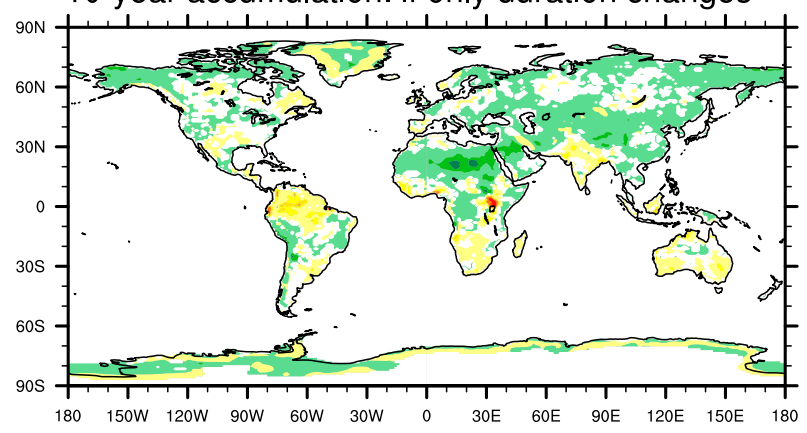

100-year accumulation: if only moisture changes

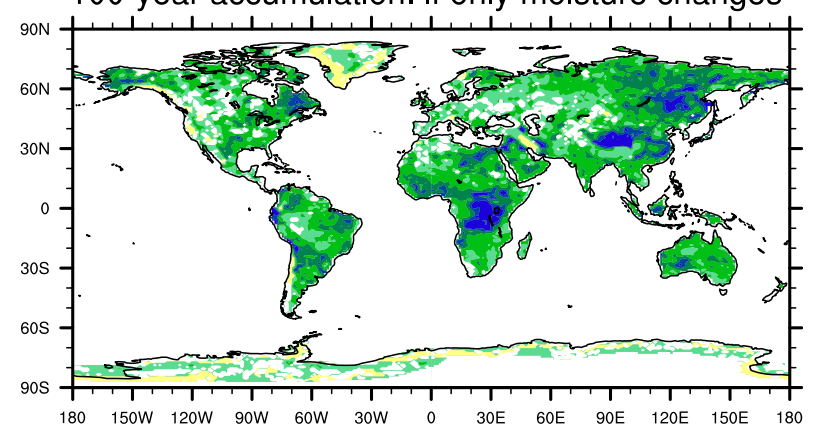

100-year accumulation: if only circulation changes

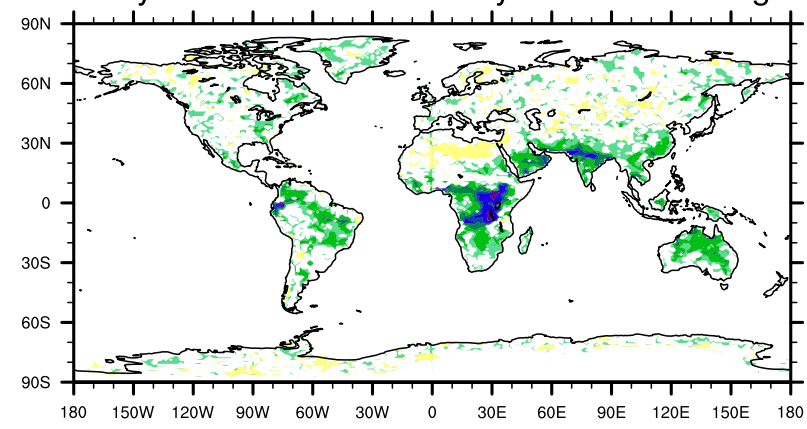

100-year accumulation: if only duration changes

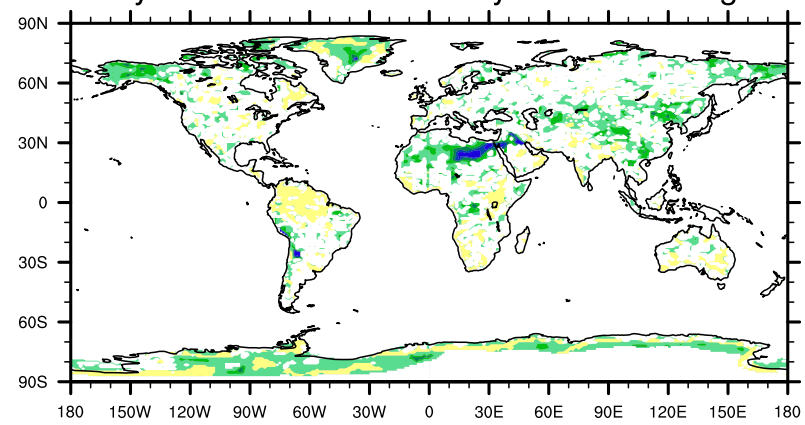

$1 / 10$

$1 / 5$

$1 / 4$

$1 / 3$

$1 / 2$

Risk ${ }^{1}$ ratio

\section{2}

3

4

5

10

FIG. 11. The risk ratio of the accumulation size which in E20 is exceeded (left) every 10 years and (right) every 100 years. The three panels in each column show how frequent the given accumulation size would become if (top) only moisture or (middle) only mass convergence or (bottom) only duration were to change, calculated by performing the analysis in Fig. 8 at each grid point. Wet and dry colors indicate an increased and decreased frequency, respectively. Values are only plotted where $>80 \%$ of bootstrap replications agree on whether the given factor increases or decreases for the 10- or 100-yr accumulation.

These results are the mean over the tropics and analysis at individual grid points reveals that in some tropical locations the positive effect due to convergence is far greater than the negative effect due to duration (Fig. 11, compare second and third rows). In particular, over China, both convergence and duration cause increases in frequency, so that the risk ratio of $P_{\text {acc }}$ for the 10- and 100-yr ARIs (Fig. 7) is far greater than due to moisture alone (Fig. 11, top panels).
In the midlatitudes, the risk ratio of $P_{\text {acc }}$ is less than due to moisture alone for low ARIs ( $<1$ year), but more than due to moisture alone for high ARIs (Fig. 10b). At low ARIs, this is due to a negative impact of convergence, that is, a weakening of circulation, but at high ARIs is due to increasing duration of events, with the 100-yr accumulation becoming 1.2 times more frequent due to duration alone. The spatial maps of risk ratio associated with duration indicate that at high ARIs the 
enhancement due to duration is predominantly in Asia and North Africa (Fig. 11, bottom row). Over these locations, there is a negative correlation with the corresponding map for convergence (Fig. 11, cf. middle and bottom rows), with global correlations over land of $r=-0.22$ for the 10 -yr accumulations and $r=-0.24$ for the 100-yr accumulations (just considering grid points with significant values in both maps). Therefore, locations where extreme events have greater convergence also have a tendency to be where duration decreases, and vice versa, which is worthy of further study. This result illustrates the importance of analyzing accumulations, as opposed to rainfall intensity-the duration factor may cancel or offset changes in rainfall rate, which the study of intensity alone does not capture. However, our results are consistent with Dwyer and O'Gorman (2017) in that changes to duration are of secondary importance for changing accumulations, with enhanced intensity (in this study represented by moisture and convergence combined) the dominant factor for changing accumulations.

\section{Summary and conclusions}

Precipitation accumulations are of fundamental importance for flooding, and their projected changes are crucial for societal adaptation to climate change. In this study, the CESM Large Ensemble projections of late twenty-first century climate were analyzed to answer the questions (i) how much more frequent will major precipitation accumulations become, and (ii) to what extent are these increases in frequency due to different factors in the moisture budget equation? In particular, how much would the frequency of major accumulations increase if only moisture, or only circulation, or only the duration of events were to change? An accumulation is defined as the integration over time of consecutive 6-hourly time slices with $P>0.5 \mathrm{~mm} \mathrm{~h}^{-1}$. Accumulations in the current (1990-2005) and projected future (2071-80) climates were sorted separately, by average recurrence interval (ARI), giving the accumulation size exceeded on average every $e$ years, with $e$ increasing exponentially from 0.1 to 100 years.

Using 6-hourly data, the accumulations for all ARIs up to 10 years are about $10 \%$ greater than using hourly data (Fig. 5), but the spatial patterns are almost identical (Fig. 6). Compared to satellite-based rainfall estimates (TRMM), CESM almost universally underestimates accumulations, particularly in the tropics (by as much as a third, but with the proportional underestimate decreasing with increasing ARI; Figs. 5 and 6). In other regions, the CESM accumulations are closer to those of TRMM (generally within about $20 \%$ ). The agreement between CESM and TRMM accumulations is best in the western United States, southern South America, India, East Asia, and Australia. Importantly, CESM and TRMM agree that there is an approximate doubling of accumulation size with each order of magnitude of ARI (e.g., the 10-yr accumulation is double the 1-yr accumulation), which is important for determining the future increase in frequency for a given accumulation size (Fig. 4).

In general, the frequency of large accumulations increases with warming, with the risk ratio, defined as the ratio of a given accumulation size's occurrence frequency between the present and future climates, increasing with ARI (Fig. 7, left panels). This results from the increase in the cutoff for large accumulations in a warmer climate (Neelin et al. 2017), so that the 100-yr event becomes more than twice as frequent almost everywhere globally. In some regions the risk ratio is particularly large, including most of the tropics and monsoon regions, where the $100-y r$ event is projected to become $>5$ times more frequent.

The dominant term in the moisture budget for accumulation size, $P_{\text {acc }}$, is the accumulated vertical moisture transport: $M_{*} C_{*} D$, where $M_{*}, C_{*}$, and $D$ represent moisture, circulation, and the duration of an event, respectively. Hence, the increased frequency of extreme accumulations can be approximated by just allowing these three factors to change in the future climate (Fig. 7, cf. left and right columns). Because $M_{*}, C_{*}$, and $D$ all increase approximately monotonically with ARI of $P_{\text {acc }}$ (Fig. 3), it is possible to relate the increased frequency of large accumulations to these factors individually.

Averaged over the tropics and midlatitudes, the increases of $P_{\text {acc }}$ due to moisture alone (about $20 \%$ at each ARI, consistent with the $3.3 \mathrm{~K}$ global-mean surface warming) are only slightly less than the actual increases in $P_{\text {acc }}$ (Fig. 8). Accordingly, the risk ratio due to moisture changes is only slightly less than the actual risk ratio of $P_{\text {acc }}$ (Fig. 10). Averaged over the tropics, the risk ratio of the 100 -yr event is 2.9 and would be 2.7 due to moisture alone (Fig. 10a). However, the contribution of enhanced circulation (increasing the magnitude of the 100-yr event by $13 \%$, averaged over the tropics) also accounts for almost a doubling of frequency of the 100-yr event, but is partly offset by a decrease due to decreasing duration (decreasing the magnitude of the 100 -yr event by $5 \%$, averaged over the tropics). These results indicate that major accumulations in the tropics will be enhanced, but also delivered in a shorter space of time, tending to enhance flood risk. Some regions are particularly vulnerable, as shown by risk ratio due to individual factors in the range of 5-10 over some tropical regions (Fig. 11). 
Averaged over the midlatitudes, the 100 -yr event becomes 2.4 times more frequent and would be 2.2 times more frequent due to moisture alone (Fig. 10b). This slight discrepancy is due to increasing duration of midlatitude events (responsible for about a $5 \%$ increase in $P_{\text {acc }}$ at all ARIs and an increase in frequency of the 100yr event by a factor of 1.2), with no changes due to circulation. The relatively small effect of duration in both the tropics and midlatitudes indicates that increasing intensity (the combination of moisture and circulation) will be by far the most important factor for increasing large precipitation accumulations, and hence determining the future increase in frequency of these major events.

Acknowledgments. GC and JN are supported by Department of Energy Grant DE-SC0016117 and National Science Foundation Grant AGS-1742178 and DN by AGS-1540518. We would like to acknowledge highperformance computing support from Cheyenne (doi: 10.5065/D6RX99HX) provided by NCAR's Computational and Information Systems Laboratory, sponsored by the National Science Foundation.

\section{APPENDIX}

\section{Additional Methods and Analyses}

\section{a. Choice of average recurrence interval for sorting accumulations}

The ARI calculated in this study contrasts with the return period based on extreme-value theory (Coles 2001). The extreme-value return period is calculated by first calculating the maximum over some interval (e.g., the annual maximum each year during the data period), combining the maxima from each interval, and using distribution fitting when there are insufficient data. The reason for using the ARI method as described in section 2 is threefold. First, large accumulations that are not necessarily the greatest in a given interval are of interest. Second, the PDF for accumulations has underlying theory that can aid understanding, hence such structure would be obscured in an extreme value PDF. Third, we wish to increase the sample size for each ARI when calculating the moisture budget over precipitating events, which we do by averaging over the probability distribution of precipitation accumulation between the $e_{i-1 / 2}$-yr and $e_{i+1 / 2}$-yr ARIs to calculate the $e_{i}$-yr accumulation (section $2 \mathrm{c}$ ). For the extreme-value returnperiod method, using just one event per year would severely restrict the number of events used when calculating the moisture budget for 100-yr events and lead to noisy results. This would also lead to more missing data. As described in section 2c, a grid point has missing data for the $i$ th ARI where there are fewer than $Y / 10^{-1+0.25(i-0.5)}$ accumulations in the data, where $Y$ is the total number of years in the aggregated dataset. In situations where there are more than $Y / 10^{-1+0.25(i-0.5)}$ accumulations, but not every interval of $10^{-1+0.25(i-0.5)}$ years contains an accumulation, there will be missing data for the return-period method, but not for the ARI method.

\section{b. Sensitivity of results to grid point aggregation}

To check that the aggregation over $3 \times 3$ grid points does not produce spurious results, the accumulations were also calculated without this spatial aggregation. The resulting spatial maps of accumulation size at each ARI are qualitatively the same, but with slightly greater accumulations at some locations when spatially aggregating (Fig. S2). This is because the spatial aggregation identifies greater accumulations at some grid point if that grid point has a wetter neighboring grid point. However, averaged over the globe, the differences are marginal; the only noticeable difference is greater smoothness in the future changes using the spatial aggregation (not shown). Therefore, the spatially aggregated results are presented in the main body of the manuscript.

\section{c. Sensitivity of results to minimum precipitation rate}

To investigate sensitivity of results to the minimum precipitation rate used to calculate accumulations, particularly with regard to duration, accumulations corresponding to the same ARIs were also calculated with an otherwise-identical method using a threshold of $0.1 \mathrm{~mm} \mathrm{~h}^{-1}$ (the threshold method is described in section $2 b$ ). In this case, accumulations at various ARIs are about $20 \%$ greater in the mid- to high latitudes and about double in the tropics, compared to the accumulations using the $0.5 \mathrm{~mm} \mathrm{~h}^{-1}$ threshold (Fig. S3). This increase results from greater duration of events, about double duration in the mid- to high latitudes and 3-4 times the duration in the tropics (Fig. S4). The smaller proportional increase in accumulation than duration using the alternative threshold indicates that the intensity of events is less using the 0.1 than $0.5 \mathrm{~mm} \mathrm{~h}^{-1}$ threshold when averaged over the much longer events.

We find that the projected risk ratio of accumulations is almost exactly the same as with the $0.5 \mathrm{~mm} \mathrm{~h}^{-1}$ threshold outside of the tropics. In the tropics, however, the risk ratio with the $0.1 \mathrm{~mm} \mathrm{~h}^{-1}$ threshold is about half that with the $0.5 \mathrm{~mm} \mathrm{~h}^{-1}$ threshold (Fig. S5). We also find that the projected future change to duration is slightly less using the 0.1 than $0.5 \mathrm{~mm} \mathrm{~h}^{-1}$ threshold, but with general agreement in sign of duration changes at each ARI (Fig. S6). 
We present the analysis using the $0.5 \mathrm{~mm} \mathrm{~h}^{-1}$ threshold because of the overrepresentation of low rain rates in climate models (Sun et al. 2006). This bias indicates that, particularly in the tropics, there are events which should be considered as distinct, but which are connected by some period of drizzle, so that the $0.1 \mathrm{~mm} \mathrm{~h}^{-1}$ threshold combines them into one event, thus greatly increasing the duration but reducing the mean intensity. We argue that the durations of events with the lower threshold (several hundreds of hours; Fig. S4) are highly unrealistic, and this issue would be even more pronounced if using no threshold, that is, accumulations consist of any precipitation $>0 \mathrm{~mm} \mathrm{~h}^{-1}$. The $0.5 \mathrm{~mm} \mathrm{~h}^{-1}$ threshold is intended to avoid this issue.

\section{d. Significance testing for projected changes}

To establish significance of the projected changes at a given ARI, bootstrapping methods were employed. For gridded figures, the analysis described above was performed for 1000 bootstrap replications. Each replication repeated the analysis described in section 2, that is, aggregating 42 members for E20 and 40 members for E21, and aggregating over $3 \times 3$ grid points, but with random sampling with replacement to choose each member. This method allows for a given replication that the same member be selected multiple times and some members not be selected at all. Significance was established if $80 \%$ of the replications agreed on the sign of the change at a given ARI. Similarly, the moisture-budget analysis in section 3 was repeated for each replication to establish significance for changes to $M_{*}^{e}, C_{*}^{e}$, and $D^{e}$. For line plots, obtained by averaging across grid points at a given ARI, the same line plot was produced using each bootstrap replication, and the full spread of a given curve across replications was plotted.

\section{e. Evaluating the moisture budget}

The simplified accumulated moisture budget equation for a given ARI, in which we state that accumulated precipitation is approximately equal to accumulated vertical moisture transport $\left(P_{\mathrm{acc}}^{e} \approx M_{*}^{e} C_{*}^{e} D^{e}\right)$ is evaluated in Fig. S7 for the 1- (top row), 10- (middle row), and 100 -yr (bottom row) accumulations. The two sides of the approximation are spatially correlated over land at $r=$ $0.90,0.89$, and 0.89 for the $1-, 10-$, and $100-$ yr accumulations, and for the same accumulations the global-mean $M_{*}^{e} C_{*}^{e} D^{e}$ over land is $83 \%, 81 \%$, and $80 \%$ of the globalmean $P_{\text {acc }}^{e}$ over land. The agreement in magnitude is best in the tropics and poorest in the mid- to high latitudes (most clearly shown by the line plots on the right). This is because, in the tropics, the horizontal advection term [the second term on the right-hand side of Eq. (3)] is negligible, similarly to the equivalent analysis for high percentiles of precipitation rate (Norris et al. 2019). For frontal events, however, advection is not negligible. To illustrate this point, the advection term $-D^{e}\left\{\overline{\mathbf{v} \cdot \nabla q\}^{e}}{ }^{e}\right.$ is added to the vertical transport term $M_{*}^{e} C_{*}^{e} D^{e}$ in Fig. S8, providing a closer approximation to $P_{\mathrm{acc}}^{e}$. The spatial correlations over land of the two sides of the approximation are increased to $r=0.97,0.97$, and 0.97 for the 1 -, $10-$, and 100-yr accumulations, and for the same accumulations the global-mean $M_{*}^{e} C_{*}^{e} D^{e}-D^{e} \overline{\{\mathbf{v} \cdot \nabla q\}^{e}}$ over land is $85 \%, 91 \%$, and $92 \%$ of the global-mean $P_{\text {acc }}^{e}$ over land. We also show a comparison between $M_{*}^{e} C_{*}^{e} D^{e}$ and $-D^{e} \overline{\{\mathbf{v} \cdot \nabla q\}^{e}}$ for the 1-, 10-, and 100-yr accumulations, illustrating the primary importance of $M_{*}^{e} C_{*}^{e} D^{e}$, but that $-D^{e} \overline{\{\mathbf{v} \cdot \nabla q\}^{e}}$ is not negligible in the midlatitudes (Fig. S9). The importance of moisture gradients over land for changes to mean precipitation was quantified by Byrne and O'Gorman (2015), implying that horizontal advection is a significant term in the moisture budget. A comparison between future changes to the two terms, that is, comparing $\Delta\left(M_{*}^{e} C_{*}^{e} D^{e}\right)$ and $-\Delta\left(D^{e} \overline{\{\mathbf{v} \cdot \nabla q\}^{e}}\right)$, reveals that the negative horizontal-advection term slightly offsets the positive vertical transport term (Fig. S10), so that future increases of accumulations are slightly less than predicted by the vertical transport term alone.

Our results indicate that, for large precipitation accumulations, the advection term is important in the mid- to high latitudes over land, and that the vertical transport term, which was decomposed in this study, does not constitute the full moisture budget. Therefore, the results in this study contain the caveat that outside of the tropics this is not the only important term.

\section{REFERENCES}

Alexander, L. V., and Coauthors, 2006: Global observed changes in daily climate extremes of temperature and precipitation. J. Geophys. Res., 111, D05109, http://dx.doi.org/10.1029/ 2005JD006290.

Allen, M. R., and W. J. Ingram, 2002: Constraints on future changes in climate and the hydrological cycle. Nature, 419, 228-232, https://doi.org/10.1038/nature01092.

Berg, P., C. Moseley, and J. O. Haerter, 2013: Strong increase in convective precipitation in response to higher temperatures. Nat. Geosci., 6, 181-185, https://doi.org/10.1038/ngeo1731.

Byrne, M. P., and P. A. O'Gorman, 2015: The response of precipitation minus evapotranspiration to climate warming: Why the "wet-get-wetter, dry-get-drier" scaling does not hold over land. J. Climate, 28, 8078-8092, https://doi.org/10.1175/JCLID-15-0369.1.

Camargo, S. J., and A. A. Wing, 2016: Tropical cyclones in climate models. Wiley Interdiscip. Rev.: Climate Change, 7, 211-237, https://doi.org/10.1002/wcc.373.

Chen, G., J. Norris, J. D. Neelin, J. Lu, L. R. Leung, and K. Sakaguchi, 2019: Thermodynamic and dynamic mechanisms for hydrological cycle intensification over the full 
probability distribution of precipitation events. J. Atmos. Sci., 76, 497-516, https://doi.org/10.1175/JAS-D-18-0067.1.

Chou, C., C.-A. Chen, P.-H. Tan, and K. T. Chen, 2012: Mechanisms for global warming impacts on precipitation frequency and intensity. J. Climate, 25, 3291-3306, https://doi.org/ 10.1175/JCLI-D-11-00239.1.

Coles, S., 2001: An Introduction to Statistical Modeling of Extreme Values. Springer, 224 pp.

Creese, A., and R. Washington, 2018: A process-based assessment of CMIP5 rainfall in the Congo basin: The SeptemberNovember rainy season. J. Climate, 31, 7417-7439, https:// doi.org/10.1175/JCLI-D-17-0818.1.

Donat, M. G., A. L. Lowry, L. V. Alexander, P. A. O'Gorman, and N. Maher, 2016: More extreme precipitation in the world's dry and wet regions. Nat. Climate Change, 6, 508-513, https:// doi.org/10.1038/nclimate2941.

Dwyer, J. G., and P. A. O'Gorman, 2017: Changing duration and spatial extent of midlatitude precipitation extremes across different climates. Geophys. Res. Lett., 44, 5863-5871, https:// doi.org/10.1002/2017GL072855.

Emori, S., and S. J. Brown, 2005: Dynamic and thermodynamic changes in mean and extreme precipitation under changed climate. Geophys. Res. Lett., 32, L17706, https://doi.org/ 10.1029/2005GL023272.

Huffman, G. J., D. T. Bolvin, E. J. Nelkin, and D. B. Wolff, 2007: The TRMM multisatellite precipitation analysis (TMPA): Quasi-global, multiyear, combined-sensor precipitation estimates at fine scales. J. Hydrometeor., 8, 38-55, https://doi.org/ 10.1175/JHM560.1.

Kao, S.-C., and A. R. Ganguly, 2011: Intensity, duration, and frequency of precipitation extremes under 21st-century warming scenarios. J. Geophys. Res., 116, D16119, https://doi.org/ 10.1029/2010JD015529.

Kay, J. E., and Coauthors, 2015: The Community Earth System Model (CESM) Large Ensemble Project: A community resource for studying climate change in the presence of internal climate variability. Bull. Amer. Meteor. Soc., 96, 1333-1349, https://doi.org/10.1175/BAMS-D-13-00255.1.

Kharin, V. V., F. W. Zwiers, X. Zhang, and M. Wehner, 2013: Changes in temperature and precipitation extremes in the CMIP5 ensemble. Climatic Change, 119, 345-357, https:// doi.org/10.1007/s10584-013-0705-8.

Martinez-Villalobos, C., and J. D. Neelin, 2018: Shifts in precipitation accumulation extremes during the warm season over the United States. Geophys. Res. Lett., 45, 8586-8595, https://doi.org/10.1029/2018gl078465.

Martins, G., C. von Randow, G. Sampaio, and A. J. Dolman, 2015: Precipitation in the Amazon and its relationship with moisture transport and tropical Pacific and Atlantic SST from the CMIP5 simulation. Hydrol. Earth Syst. Sci. Discuss., 12, 671704, https://doi.org/10.5194/hessd-12-671-2015.

Neelin, J. D., S. Sahany, S. N. Stechmann, and D. N. Bernstein, 2017: Global warming precipitation accumulation increases above the current-climate cutoff scale. Proc. Natl. Acad. Sci. USA, 114, 1258-1263, https://doi.org/10.1073/ pnas. 1615333114.

Norris, J., G. Chen, and J. D. Neelin, 2019: Thermodynamic versus dynamic controls on extreme precipitation in a warming climate from the Community Earth System Model Large
Ensemble. J. Climate, 32, 1025-1045, https://doi.org/10.1175/ JCLI-D-18-0302.1.

O'Gorman, P. A., and T. Schneider, 2009: The physical basis for increases in precipitation extremes in simulations of 21stcentury climate change. Proc. Natl. Acad. Sci. USA, 106, 14 773-14 777, https://doi.org/10.1073/pnas.0907610106.

Pall, P., M. R. Allen, and D. A. Stone, 2007: Testing the ClausiusClapeyron constraint on changes in extreme precipitation under $\mathrm{CO}_{2}$ warming. Climate Dyn., 28, 351-363, https:// doi.org/10.1007/s00382-006-0180-2.

Parzybok, T. W., B. Clarke, and D. M. Hultstrand, 2011: Average recurrence interval of rainfall in real-time. World Environmental and Water Resources Congress 2011: Bearing Knowledge for Sustainability, Palm Springs, CA, ASCE, 4667-4681, https://doi.org/10.1061/41173(414)486.

Pendergrass, A. G., and D. L. Hartmann, 2014a: Changes in the distribution of rain frequency and intensity in response to global warming. J. Climate, 27, 8372-8383, https://doi.org/ 10.1175/JCLI-D-14-00183.1.

- , and $-2014 \mathrm{~b}$ : Two modes of change of the distribution of rain. J. Climate, 27, 8357-8371, https://doi.org/10.1175/JCLID-14-00182.1.

_ R. Knutti, F. Lehner, C. Deser, and B. M. Sanderson, 2017: Precipitation variability increases in a warmer climate. Sci. Rep., 7, 17966, https://doi.org/10.1038/s41598-017-17966-y.

Peters, O., A. Deluca, A. Corral, J. D. Neelin, and C. E. Holloway, 2010: Universality of rain event size distributions. J. Stat. Mech., 2010, P11030, https://doi.org/10.1088/1742-5468/2010/ 11/P11030.

Pfahl, S., P. A. O'Gorman, and E. M. Fischer, 2017: Understanding the regional pattern of projected future changes in extreme precipitation. Nat. Climate Change, 7, 423-428, https://doi.org/ 10.1038/nclimate3287.

Seager, R., and N. Henderson, 2013: Diagnostic computation of moisture budgets in the ERA-Interim reanalysis with reference to analysis of CMIP-archived atmospheric model data. J. Climate, 26, 7876-7901, https://doi.org/10.1175/JCLI-D-1300018.1.

Sillmann, J., V. V. Kharin, F. W. Zwiers, X. Zhang, and D. Bronaugh, 2013: Climate extremes indices in the CMIP5 multimodel ensemble: Part 2. Future climate projections. J. Geophys. Res. Atmos., 118, 2473-2493, https://doi.org/ 10.1002/JGRD.50188.

Stechmann, S. N., and J. D. Neelin, 2014: First-passage-time prototypes for precipitation statistics. J. Atmos. Sci., 71, 32693291, https://doi.org/10.1175/JAS-D-13-0268.1.

Sun, Y., S. Solomon, A. Dai, and R. W. Portmann, 2006: How often does it rain? J. Climate, 19, 916-934, https://doi.org/10.1175/ JCLI3672.1.

Tandon, N. F., X. Zhang, and A. H. Sobel, 2018: Understanding the dynamics of future changes in extreme precipitation intensity. Geophys. Res. Lett., 45, 2870-2878, https://doi.org/10.1002/ 2017GL076361.

Trenberth, K. E., 1999: Conceptual framework for changes of extremes of the hydrological cycle with climate change. Climatic Change, 42, 327-339, https://doi.org/10.1023/A:1005488920935.

Westra, S., L. V. Alexander, and F. W. Zwiers, 2013: Global increasing trends in annual maximum daily precipitation. J. Climate, $\mathbf{2 6}$, 3904-3918, https://doi.org/10.1175/JCLI-D-12-00502.1. 